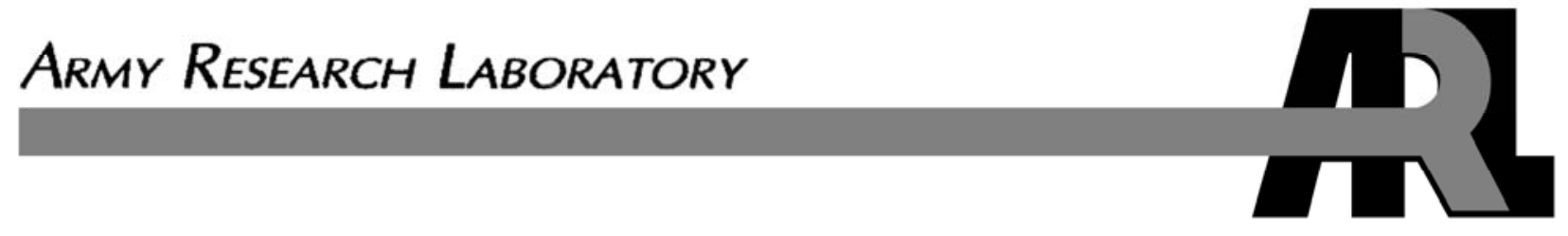

\title{
Mechano-Chemical Stability of Gold Nanoparticles Coated With Alkanethiolate SAMs
}

\author{
by Brian J. Henz, Takumi Hawa, and Michael R. Zachariah
}




\section{NOTICES}

\section{Disclaimers}

The findings in this report are not to be construed as an official Department of the Army position unless so designated by other authorized documents.

Citation of manufacturer's or trade names does not constitute an official endorsement or approval of the use thereof.

Destroy this report when it is no longer needed. Do not return it to the originator. 


\title{
Army Research Laboratory
}

Aberdeen Proving Ground, MD 21005-5067

ARL-TR-5048

December 2009

\section{Mechano-Chemical Stability of Gold Nanoparticles Coated With Alkanethiolate SAMs}

\author{
Brian J. Henz \\ Computational and Information Sciences Directorate, ARL \\ Takumi Hawa and Michael R. Zachariah \\ University of Maryland, College Park \\ and \\ National Institute of Standards and Technology
}




\section{REPORT DOCUMENTATION PAGE}

Form Approved OMB No. 0704-0188

Public reporting burden for this collection of information is estimated to average 1 hour per response, including the time for reviewing instructions, searching existing data sources, gathering and maintaining the data needed, and completing and reviewing the collection information. Send comments regarding this burden estimate or any other aspect of this collection of information, including suggestions for reducing the burden, to Department of Defense, Washington Headquarters Services, Directorate for Information Operations and Reports (0704-0188), 1215 Jefferson Davis Highway, Suite 1204, Arlington, VA 22202-4302. Respondents should be aware that notwithstanding any other provision of law, no person shall be subject to any penalty for failing to comply with a collection of information if it does not display a currently valid OMB control number.

PLEASE DO NOT RETURN YOUR FORM TO THE ABOVE ADDRESS.

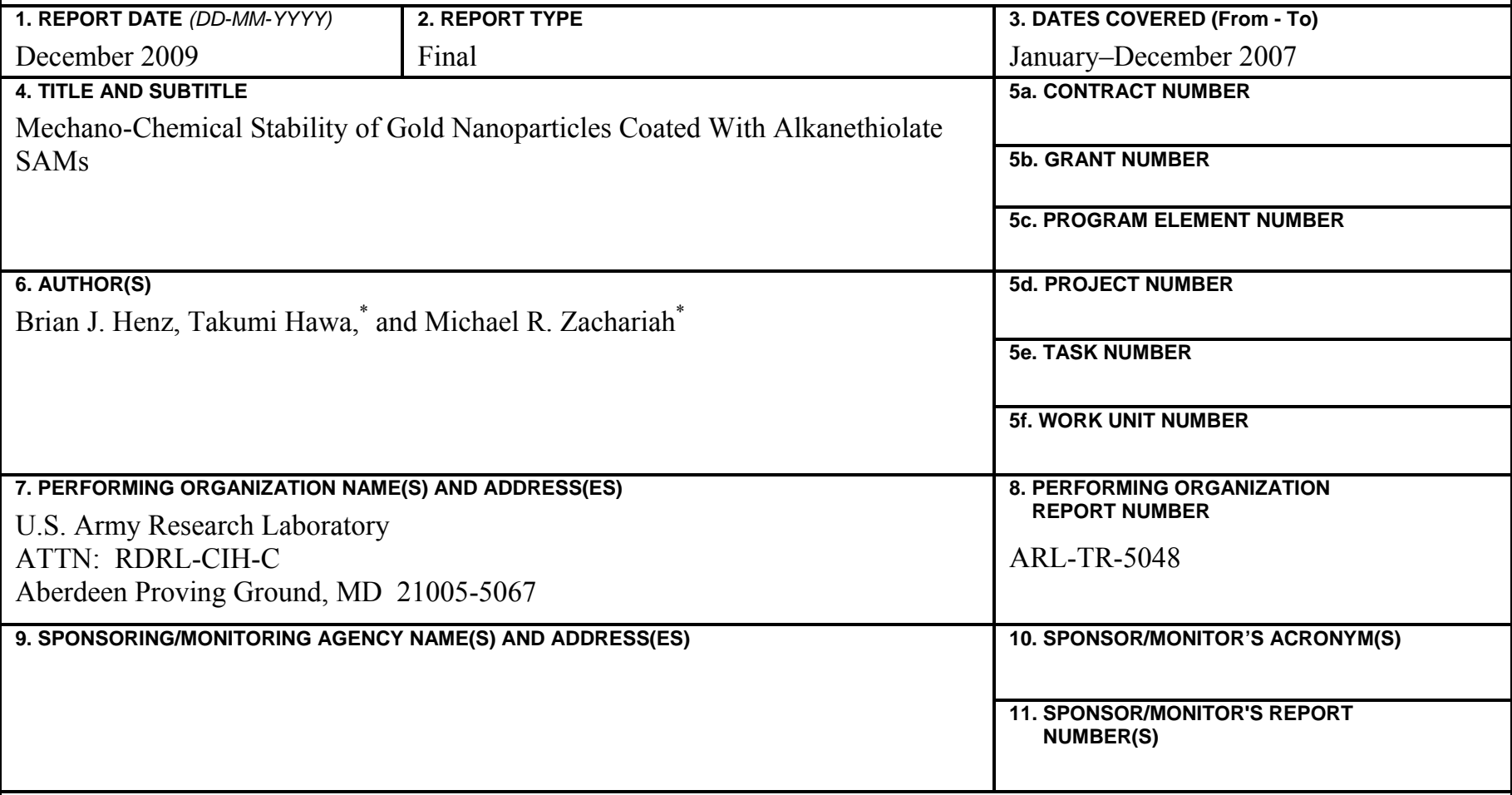

\section{DISTRIBUTIONIAVAILABILITY STATEMENT}

Approved for public release; distribution is unlimited.

\section{SUPPLEMENTARY NOTES}

*Department of Mechanical Engineering and the Department of Chemistry and Biochemistry, University of Maryland, College Park; National Institute of Standards and Technology, Gaithersburg, MD

\section{ABSTRACT}

Molecular dynamics simulations are used to probe the structure and stability of alkanethiolate self-assembled monolayers (SAMs) on gold nanoparticles. We have observed that the surface of gold nanoparticles becomes highly corrugated by the adsorption of the SAMs. Furthermore, as the temperature is increased, the SAMs dissolve into the gold nanoparticle, creating a liquid mixture at temperatures much lower than the melting temperature of the gold nanoparticle. By analyzing the mechanical and chemical properties of gold nanoparticles at temperatures below the melting point of gold, with different SAM chain lengths and surface coverage properties, we have determined that the system is metastable. The model and computational results that provide support for this hypothesis are presented.

\section{SUBJECT TERMS}

molecular dynamics, nanoparticles, self-assembled monolayer

\begin{tabular}{|l|l|l|c|c|l|}
\hline \multicolumn{2}{|l|}{ 16. SECURITY CLASSIFICATION OF: } & $\begin{array}{c}\text { 17. LIMITATION } \\
\text { OF ABSTRACT }\end{array}$ & $\begin{array}{l}\text { 18. NUMBER } \\
\text { OF PAGES }\end{array}$ & $\begin{array}{l}\text { 19a. NAME OF RESPONSIBLE PERSON } \\
\text { Brian J. Henz }\end{array}$ \\
\cline { 1 - 2 } $\begin{array}{l}\text { a. REPORT } \\
\text { Unclassified }\end{array}$ & $\begin{array}{l}\text { b. ABSTRACT } \\
\text { Unclassified }\end{array}$ & $\begin{array}{l}\text { c. THIS PAGE } \\
\text { Unclassified }\end{array}$ & UU & 40 & $\begin{array}{l}\text { 19b. TELEPHONE NUMBER (Include area code) } \\
410-278-6531\end{array}$ \\
\hline
\end{tabular}




\section{Contents}

List of Figures $\quad$ iv

$\begin{array}{ll}\text { Acknowledgments } & \text { v }\end{array}$

1. Introduction and Background $\quad 1$

2. Computational Modeling and Numerical Procedure 2

3. Simulating the Gold Substrate $\quad 3$

4. Modeling the Alkanethiolate Chain $\quad 3$

5. Binding Sulfur to Gold $\quad 4$

6. Simulation Results $\quad 5$

7. Pair Correlation Function $\quad 6$

$\begin{array}{lr}\text { 8. Diffusion } & 8\end{array}$

9. Radial Pressure Distribution $\quad 11$

10. Radial Density Distribution $\quad 16$

11. Phase Behavior and SAM Solubility $\quad 19$

12. SAM Phases $\quad 22$

$\begin{array}{lr}\text { 13. Conclusions } & 24\end{array}$

14. References $\quad 26$

$\begin{array}{ll}\text { Distribution List } & 31\end{array}$ 


\section{List of Figures}

Figure 1. Sample alkanethiolate chain with eight carbon atoms along the backbone. 4

Figure 2. (a) Pair correlation function for bare 50 - $\AA$ gold nanoparticle. (b) Pair correlation function results for the gold atoms in a 50- $\AA$ gold nanoparticle coated with an alkanethiolate SAM at various temperatures with a sulfur binding energy of $184 \mathrm{~kJ} / \mathrm{mol}$. (c) Pair correlation function results for the SAM-coated gold nanoparticle with the lower sulfur binding energy of $126 \mathrm{~kJ} / \mathrm{mol}$. (d) Comparison of the pair correlation results at 500 $\mathrm{K}$ for the bare nanoparticle and high $(184 \mathrm{~kJ} / \mathrm{mol})$ and low $(126 \mathrm{~kJ} / \mathrm{mol})$ alkanethiol binding energies.

Figure 3. (a) Arrhenius plot of diffusivity for sulfur atoms. The activation energy for SAM mobility is estimated by the slope of $\ln (D)$ vs. $1 / T$ between 100 and $800 \mathrm{~K}$. (b) Ratio of sulfur diffusivity to gold diffusivity in the alkanethiol-coated gold nanoparticle material system.

Figure 4. Radial pressure distribution for 50 - $\AA$ gold nanoparticle at various temperatures.........11

Figure 5. Radial pressure distribution for a 50- $\AA$ gold nanoparticle with alkanethiolate SAMs with differing interaction coefficients.

Figure 6. Radial pressure distribution for a fully coated gold nanoparticle for range of temperatures with high binding energy $(184.1 \mathrm{~kJ} / \mathrm{mol})$.

Figure 7. Radial pressure distribution for alkanethiol-coated $50-\AA \AA$ gold nanoparticle with $126 \mathrm{~kJ} / \mathrm{mol}$ sulfur-gold binding energy.

Figure 8. Radial density of gold in alkanethiolate SAM-coated gold nanoparticle for various surface coverage amounts.

Figure 9. PE vs. temperature for the alkanethiolate SAM-coated gold nanoparticle with various amount of surface coverage and the $184 \mathrm{~kJ} / \mathrm{mol}$ sulfur binding energy.

Figure 10. Cross section of 100\% coated gold nanoparticle at (a) $300 \mathrm{~K}$ and (b) $600 \mathrm{~K}$, showing the dissolution of the alkanethiol chains at high temperatures. (The blue spheres represent gold atoms, light blue are sulfur, yellow are $\mathrm{CH}_{2}$, and red are $\mathrm{CH}_{3}$.) ....

Figure 11. (a) PE vs. temperature for the alkanethiolate SAM-coated gold nanoparticle with various amounts of surface coverage and $126 \mathrm{~kJ} / \mathrm{mol}$ binding energy. (b) Comparison of $\mathrm{PE}$ vs. temperature for fully coated nanoparticle with 184 and $126 \mathrm{~kJ} / \mathrm{mol}$ binding energy...21

Figure 12. Plot of specific heat vs. surface coverage for alkanethiolate SAM-coated gold nanoparticle showing exponential relationship between specific heat and surface coverage.

Figure 13. Difference in radial position for the head and tail groups of the alkanethiolate chains at various surface coverage densities, (a) drawing and (b) tilt angle ( $\theta$ ). The alkanethiol radius is also labeled as the monolayer thickness.

Figure 14. Drawing showing the difference in space occupied by tail molecule on curved and flat gold surfaces, assuming a chain length of nine carbon atoms. 


\section{Acknowledgments}

The authors would like to acknowledge the support received by the U.S. Army Research Laboratory Department of Defense Supercomputing Resource Center at the Aberdeen Proving Ground, MD. Additional support was given by the National Institute for Standards Technology. 
INTENTIONALLY LEFT BLANK. 


\section{Introduction and Background}

Nanoparticles are often used in applications where a high surface-area-to-volume ratio is desired. Some typical applications include catalysis, biosensors, and use as drug delivery vehicles $(1,2)$. A major limitation of the expanded use of these nanoparticles is that the fabrication of nanoparticles with the desired morphology and structure is challenging (3-5). Gold is a commonly used material because of its resistance to oxidation and its interesting electrical, magnetic, optical, and physical properties ( 6 ). Furthermore, there is a considerable knowledge base on functionalizing gold surfaces that are being ported to analogous particles (7). For this reason, gold nanoparticles are commonly chosen for basic research because of the availability of experimental $(8,9)$ and atomistic simulation data $(10,11)$. For instance, using x-ray powder diffraction, Cleveland et al. (9) discovered that small 10- to 20- $\AA$-diameter gold nanoparticles form a truncated-decahedral motif geometry. Using molecular dynamics (MD) simulations, Lewis et al. (12) were able to determine the melting process for gold nanoparticles. This last result is important because the authors found that the surface atoms melt first and at a temperature below that of the atoms in the core of the nanoparticle. The implications of this for coated nanoparticles are that the surface atoms, where binding of the monolayer takes place, are affected by a lower temperature than is expected from the melting temperature of the whole nanoparticle. The results of these past efforts are built upon in this work in order to analyze in larger gold nanoparticles the nature and role of surface functionalization.

Coating of gold surfaces and nanoparticles with a self-assembled monolayer (SAM) creates many additional applications, including lithography, lubrication, catalysis, biocompatible materials, and biosensors $(6,13)$. Numerous experimental $(14-16)$ and numerical $(13,17,18)$ studies have attempted to characterize the properties of alkanethiolate SAM-coated gold surfaces. Simulations investigating alkanethiolate SAM-coated gold surfaces accurately predict the $\mathrm{c}(4 \times 2)$ superlattice structure of alkanethiols on an $\mathrm{Au}(111)$ surface (17). Additional work has predicted the segregation of SAM chains adsorbed on a gold surface by length (13), surface frictional forces (19), and phase behavior (20). Efforts to characterize the alkanethiolate SAM coated gold nanoparticle system have been limited to atomistic simulations considering a single alkanethiol chain and a small $(<100$ atoms) gold cluster $(17,21)$ or MD simulations of small nanoparticles that ignore gold-gold interactions $(6,18)$.

During the fabrication of nanoparticles, the propensity to agglomerate and sinter must be inhibited (18) by the passivation of the nanoparticle surface. The use of coatings for surface passivation has been found to significantly reduce the degree to which agglomeration and sintering will occur (22). For instance, Hawa and Zachariah found that a hydrogen surface passivation coating used with a silicon nanoparticle will prevent reaction with other particles 
when collided at thermal velocities (22) or will retard the process (23). Other effects related to the passivation layer investigated computationally include changes in the internal pressure and surface tension (24).

This report is focused on understanding the effect of the alkanethiolate SAM on gold nanoparticles using MD simulations. Using the pair correlation function, we estimate the melting temperature of the particle and the effect that the SAM has on the particle surface. We clarify the relationship between surface coverage and particle structure by introducing a corrugation factor, and identify the phase transition mechanism of SAM-coated gold nanoparticles. We find that the SAM-coated particles are mechanically unstable as determined by the radial pressure profile.

\section{Computational Modeling and Numerical Procedure}

There are numerous documented MD studies of alkanethiolate SAMs on gold surfaces $(13,17$, $19)$. However, these studies have been limited to flat gold surfaces or to fixed gold atoms in a nanocrystallite $(6,18)$. In addition to, and in support of, these previous MD simulations, ab initio quantum chemical calculations have been used to simulate gold clusters with short alkanethiolate SAMs, primarily to determine potential parameters for MD or Monte Carlo (MC) computations. The ab initio calculations show that the gold crystal lattice is perturbed by the adsorbed ligand (25). The consequence of the perturbation is an increase in $\mathrm{Au}-\mathrm{Au}$ bond length of up to $20 \%$. The increase in bond length is observed around the adsorption site with relaxation propagating to the second layer. These results suggest that the MD simulations should include dynamic gold atoms in order to accurately consider the nanoparticle/SAM system.

Alkanethiol chains adsorbed on a flat surface made of immobile gold atoms are limited to diffusion in only two dimensions, whereas chains adsorbed onto a nanoparticle surface with mobile gold atoms may diffuse in three dimensions. This disparity in model detail is expected to provide more insight into the SAM-coated nanoparticle system than was previously available. The inclusion in the simulation of a surface constructed of dynamic gold atoms should be able to more accurately predict alkanethiol chain movement than the limited movement possible on a flat surface of fixed gold atoms.

The size of the model system investigated here is determined by approximating experimentally realistic systems. The manufacture of consistently sized, small-diameter nanoparticles becomes more difficult at diameters of $<50 \AA$; therefore, in this work, $50-\AA$-diameter gold nanoparticles (4093 gold atoms) with and without SAMs are considered. The SAM considered consists of alkanethiolate chains that contain a sulfur atom head group that binds to the gold surface and a carbon backbone with 3 to 18 carbon atoms comprising the alkyl chain. 
The MD simulations in this work are carried out using the Large-scale Atomic/Molecular Massively Parallel Simulator (LAMMPS) (20) software.

\section{Simulating the Gold Substrate}

In this effort, the gold substrate atoms are explicitly considered as dynamic atoms, requiring that an accurate potential be used to simulate these atomic interactions. The embedded atom method (EAM) $(11,27,28)$ and the effective medium (29-31) potentials have received the most attention for accurately simulating atomic gold interactions and the interactions of other face-centered cubic (FCC) lattice metals. Both methods use empirical embedding functions that originate from density functional theory and have been successfully used to model many of the physical properties of gold. We chose the EAM for modeling the gold substrate because of its reported accuracy and the potentially important extensions developed for the EAM for modeling surfaces, such as the extended EAM (32) and the surface EAM (33). The actual EAM potential used in this work is taken from LAMMPS (26). Also considered was a second dataset from Chen et al. (34). A detailed discussion of the method as used in this work can be found in Daw and Baskes (11) and Foiles et al. (27).

\section{Modeling the Alkanethiolate Chain}

The alkanethiolate polymer chain may be simulated with a range of computational complexity depending upon the resources available and the accuracy of results required. For MD simulations, the three methods available for modeling the alkanethiol chain are the highaccuracy/high-computational requirement all-atom model, the less accurate/lower-computational requirement united atom method, and the coarse-grained bond model. These methods can be compared by considering the $\mathrm{CH}_{2}$ molecule that makes up the backbone of the alkanethiolate chain. When the united atom method is used, only one particle is simulated per backbone monomer, as opposed to three particles with an all-atom simulation (35). Finally, with the coarse-grained model, multiple molecules are clustered together into one particle with perhaps five or more $\mathrm{CH}_{2}$ molecules grouped into one simulated particle (36-38). For the simulations in this work, we have chosen the united atom method, which is computationally more efficient than the all-atom method yet provides sufficient accuracy for the current analysis (18).

The modeling of the alkanethiol chain using the united atom model is consistent with many of the published studies for the alkanethiolate-coated gold nanoparticle system $(6,17,18,20)$. An example of the alkanethiolate polymer chain model used in this work is shown in figure 1 . The 


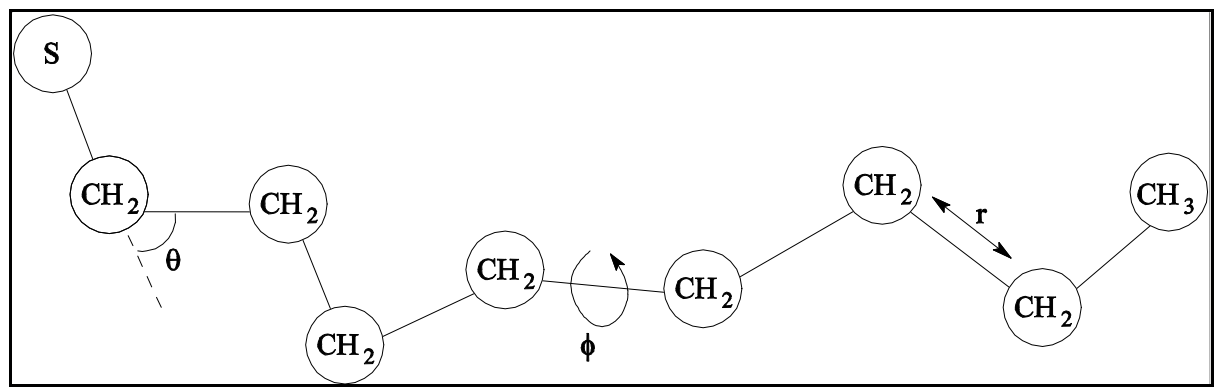

Figure 1. Sample alkanethiolate chain with eight carbon atoms along the backbone.

potentials used in the united atom model include a bending potential for the S-C-C bond and the $\mathrm{C}-\mathrm{C}-\mathrm{C}$ bond. Bond bending can be modeled as either a flexible bond or a stiff bond where the angle is held constant. There is a dihedral potential for the $\mathrm{X}-\mathrm{C}-\mathrm{C}-\mathrm{X}$ bonds, where $\mathrm{X}$ can be either $\mathrm{S}$ or $\mathrm{C}$. There are two common methods used in the literature to model the bond lengths along the chain. The bond lengths along the chain can be held constant (13) with the RATTLE (39) algorithm, or they can be modeled as harmonic bonds $(40,41)$. Using a harmonic bond to describe the $\mathrm{C}-\mathrm{C}$ and $\mathrm{S}-\mathrm{C}$ backbone bonds allows the distance between the backbone atoms to change while not allowing the bonds to break during the simulation.

The potentials and parameter values for the bending angle and dihedral angle from figure 1 and used in this report are given in Shevade et al. (13); the bond stretch parameters are given in Rai et al. (40).

\section{Binding Sulfur to Gold}

The binding potential of the head sulfur atom of an alkanethiol chain to the gold substrate is of great interest as it affects the location, orientation, movement, and desorption of chains from the gold surface $(6,41,42)$. Much effort has been devoted to finding accurate potentials for simulating the sulfur-to-gold binding energy from ab initio methods $(6,42)$. The most commonly published potentials resulting from these calculations are the 12-3 potential (43), used most often in MC simulations of alkanethiolates on gold $(13,19)$, and the Morse potential, used in many of the MD simulation studies $(19,17,41,44)$. In addition to the issue of which potential best describes the binding between SAM headgroup and substrate, the possibility of SAM mobility must also be considered (42). Since the Morse potential, equation 1, can mimic a partially covalent bond (17), it is used in MD simulations along with the parameters given by Zhang et al. (17) to model the Au-S bond. The parameters for equation 1 are $D_{e}=13.3 \mathrm{~kJ} / \mathrm{mol}$, $\alpha=1.38$, and $r_{0}=2.903 \AA$.

$$
U_{\text {Morse }}(r)=D_{e}\left[e^{-2 \alpha\left(r-r_{0}\right)}-2 e^{-\alpha\left(r-r_{0}\right)}\right]
$$


The binding energy of the alkanethiol to the gold surface has been determined to be around $184.1 \mathrm{~kJ} / \mathrm{mol}$ (13) when all of the sulfur-gold interactions are considered. Although this reported value is often referenced, there are several studies that report the sulfur-gold bond energy to be closer to $126.0 \mathrm{~kJ} / \mathrm{mol}(45,46)$. This discrepancy in the binding energy is large, and so in this report we will investigate using both binding energies in order to understand the effect that binding energy has on the computed results. For the sulfur-gold binding energy of $126.0 \mathrm{~kJ} / \mathrm{mol}$, the Morse potential parameter is $D_{e}=9.108 \mathrm{~kJ} / \mathrm{mol}$.

\section{Simulation Results}

In this section, the results from the MD simulations are presented and analyzed. In order to study the effects of the alkanethiolate SAM on the gold nanoparticle, we have computed many system properties. These properties include diffusion coefficients, radial pressure and density distributions, melting temperature, and the pair correlation function. A corrugation factor that describes the depth to which the gold surface is modified by the adsorbed alkanethiol chains is also defined in this section. Each result is a data point of the changes the nanoparticle surface experiences with the adsorption of the alkanethiolate SAM. For instance, the diffusion coefficient is used to compute the mobility of the SAM chains that are adsorbed on the surface of the nanoparticle and the mobility of the gold atoms near the surface of the nanoparticle. The combination of these two coefficients will partially define the phase of the materials at and on the nanoparticle surface and also help in predicting if nucleation of the SAM chains will occur. Nucleation may occur under low surface coverage conditions when the chains form dense groups and uneven surface coverage. The density distribution is used to find the depth to which the sulfur atoms penetrate the surface of the gold nanoparticle and to compute the corrugation factor.

The simulation results will also be compared for the two sulfur-gold binding energies considered in this work. The computed results will be compared and discussed in order to better understand how the head group binding energy affects the properties of the nanoparticle and anticipated trends for these properties. It is expected that the lower binding energy will have less of an effect on the properties of the gold nanoparticle than the high binding energy. On the other hand, some processes such as desorption will possibly be observed only at the lower binding energy because the temperature required for desorption will be lower.

The simulation is initialized with a sphere of FCC lattice gold atoms at $0 \mathrm{~K}$. By applying a random velocity distribution to the gold atoms, the temperature is raised to $10 \mathrm{~K}$ and then slowly increased over $10^{7}$ time steps to $1400 \mathrm{~K}$, or above the bulk melting temperature of gold. By rescaling the velocity, the temperature is then decreased to $300 \mathrm{~K}$ over $8 \cdot 10^{6}$ time steps and then held for another $8 \cdot 10^{6}$ time steps. The temperature rescaling fix is then removed in order to determine if the total energy of the system remains constant. The nanoparticle now contains many large facets with (111) and (100) faces, as one would expect from experimental 
observations (47). The alkanethiol chains are next randomly distributed around the nanoparticle with the sulfur head group at the equilibrium distance from the gold surface. The alkanethiol chains are initially orientated radially from the nanoparticle center. The alkanethiol chains were initialized with $100 \%$ gauche defects. The effect of this initialization on the observed simulation results is negligible as many of the gauche defects are removed during equilibration. The only result that would not be considered accurate is a quantitative measurement of the fraction of gauche defects under various conditions. The number of chains is determined by the requested surface area per chain. The temperature is again initialized to $10 \mathrm{~K}$ and slowly heated to $300 \mathrm{~K}$ and then held until the total system energy remains constant, indicating that equilibrium has been achieved. The temperature during equilibration of the alkanethiol-coated nanoparticle is not allowed to increase beyond $300 \mathrm{~K}$ because desorption is expected to occur beyond this temperature (7).

\section{Pair Correlation Function}

The pair correlation function, $g(r)$, is defined as the number of atoms a distance $r$ from a given atom compared with the number of atoms at the same distance in an ideal gas at the same density (48). The pair correlation function for a bare 50 - $\AA$-diameter gold nanoparticle is shown in figure 2 for a range of temperatures.

The results in figure $2 \mathrm{a}$ indicate that a phase transition occurs in the nanoparticle from a crystalline solid at $900 \mathrm{~K}$ to a liquid at $1000 \mathrm{~K}$, and, as expected, this temperature range is below that of the bulk melting temperature of $1340 \mathrm{~K}$ (12).

For a spherical nanoparticle, the melting temperature can be crudely predicted from experimental data using equation 2 , where $\rho_{S}$ and $\rho_{L}$ are the specific mass of the solid and liquid phases, respectively. $\gamma_{S}$ and $\gamma_{L}$ are the surface energies of the solid and liquid phases, respectively, $D_{p}$ is the particle diameter, $L$ is the heat of fusion, and $T_{\infty}$ and $T\left(D_{p}\right)$ are the bulk- and sizedependent melting temperatures, respectively. Using published data for gold (12), the predicted melting temperature of a 50 - $\AA$-diameter gold nanoparticle is about $995 \mathrm{~K}$. This result is within the range of our MD simulation prediction for the melting temperature.

$$
\frac{T\left(D_{p}\right)-T_{\infty}}{T_{\infty}}=\frac{4}{\rho_{S} L D_{p}}\left[\gamma_{S}-\gamma_{L}\left(\rho_{S} / \rho_{L}\right)^{2 / 3}\right] .
$$




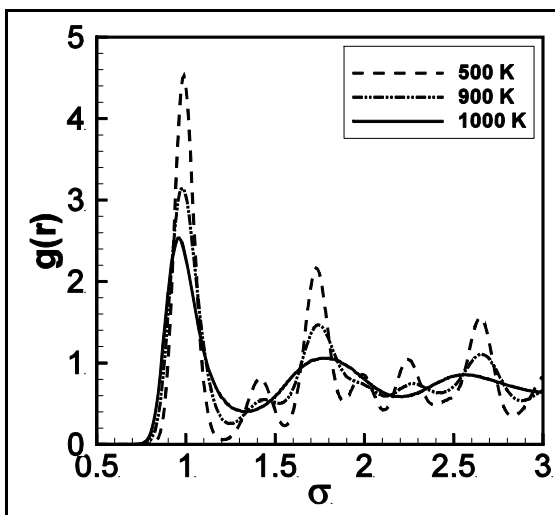

a)

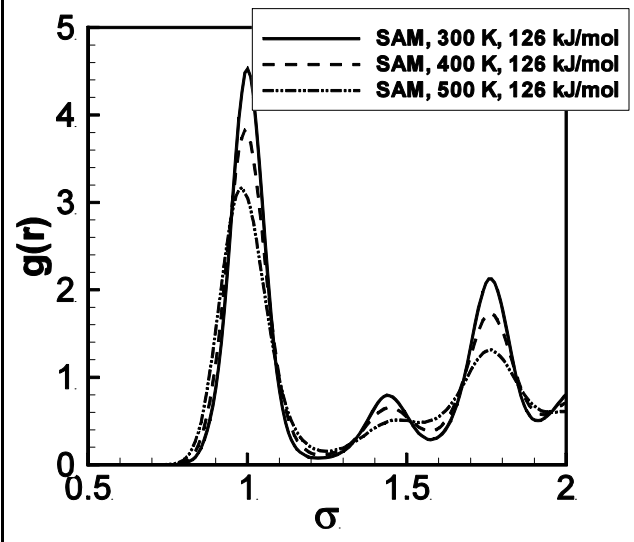

c)

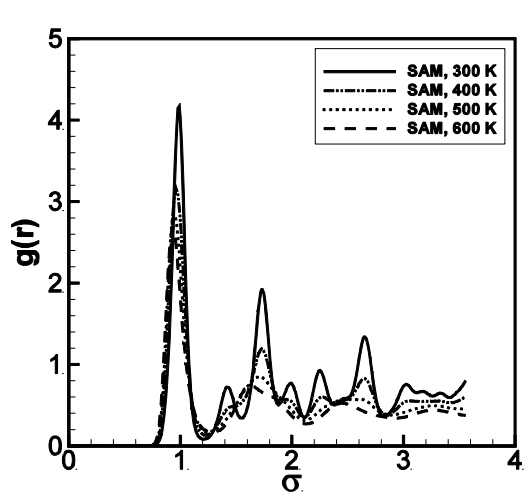

b)

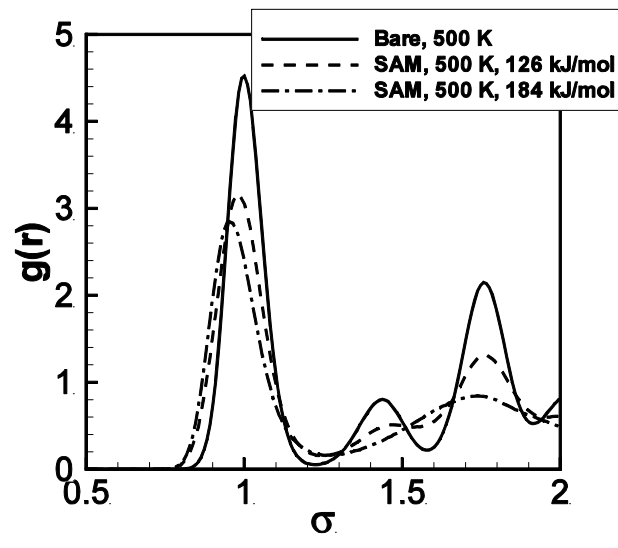

d)

Figure 2. (a) Pair correlation function for bare 50-Å gold nanoparticle. (b) Pair correlation function results for the gold atoms in a 50- $\AA$ gold nanoparticle coated with an alkanethiolate SAM at various temperatures with a sulfur binding energy of $184 \mathrm{~kJ} / \mathrm{mol}$. (c) Pair correlation function results for the SAM-coated gold nanoparticle with the lower sulfur binding energy of 126 $\mathrm{kJ} / \mathrm{mol}$. (d) Comparison of the pair correlation results at $500 \mathrm{~K}$ for the bare nanoparticle and high $(184 \mathrm{~kJ} / \mathrm{mol})$ and low $(126 \mathrm{~kJ} / \mathrm{mol})$ alkanethiol binding energies.

Next we compare the pair correlation function results for the uncoated gold nanoparticle to the results for an alkanethiolate-coated gold nanoparticle. The coating considered is a full coating, meaning one chain per $15.4 \AA^{2}$ of surface area on the gold nanoparticle (49), and is about $30 \%$ denser than the packing density on a flat surface ( $\left.21.4 \AA^{2}\right)(15)$. The pair correlation function results for the alkenthiolate SAM-coated gold nanoparticle show a nanoparticle with a crystalline structure for temperatures below $400 \mathrm{~K}$, and an amorphous structure above $600 \mathrm{~K}$ (see figure $2 \mathrm{~b}$ ). The pair correlation function is computed for the gold atoms only in figure $2 \mathrm{~b}$. By only considering the gold atoms in computing the pair correlation results, the structure of the nanoparticle is analyzed separately from the SAM. At $500 \mathrm{~K}$, the structure of the gold nanoparticle has changed to a less ordered structure, similar to that observed during the melting of the uncoated gold nanoparticle. This change in the pair correlation function 
indicates that a phase change occurs in the gold nanoparticle between 400 and $500 \mathrm{~K}$. Hence, the melting temperature of the gold nanoparticle is lowered by about $500 \mathrm{~K}$ by mixing with the alkanethiolate chains.

In the next set of simulations, we considered the lower gold-sulfur binding energy of $126 \mathrm{~kJ} / \mathrm{mol}$. In these simulations, the chain-chain interactions use the same interaction potentials discussed earlier. In figure $2 \mathrm{c}$, the pair correlation results for the fully coated gold nanoparticle are plotted for temperatures of 300,400 , and $500 \mathrm{~K}$. Notice that at $500 \mathrm{~K}$, the nanoparticle is still highly structured. This indicates that the nanoparticle does not undergo a phase change at $500 \mathrm{~K}$ as is observed in the high binding energy results. In figure $2 \mathrm{~d}$, the pair correlation results for the gold nanoparticle at $500 \mathrm{~K}$ are compared for the uncoated and coated nanoparticles with high and low binding energy. In this figure, the effect of the high binding energy is clearly evident. The lower degree of crystallinity with the higher binding energy indicates a more liquid-like structure at $500 \mathrm{~K}$.

\section{Diffusion}

It is apparent from the results of the previous section that the alkanethiolate SAM coating has an appreciable effect on the gold nanoparticle properties. In order to gain a better understanding of the SAM and how it interacts with the gold nanoparticle, we have computed the diffusion coefficient for the SAM and the gold atoms. The alkanethiolate chain is primarily bound to the gold nanoparticle through the sulfur-gold interactions at the head of the chain. The diffusion coefficient of the sulfur atoms is of particular interest because it will provide insight into the mobility of the alkanethiol chains in relation to the surface gold atoms. The diffusion coefficient of the alkanethiolate chain is calculated in the MD simulations by computing the mean squared displacement (MSD) of the sulfur head group. The diffusion coefficient, $D$, is then evaluated using equation 3.

$$
\frac{\partial\left\langle r^{2}(t)\right\rangle}{\partial t}=2 d D .
$$

In equation 3 , the number of dimensions available for atomic diffusion, $d$, is assumed to be $3, t$

is time, and $\left\langle r^{2}(t)\right\rangle$ is the MSD of the atoms being tracked. For diffusion on a surface, $d$ would typically be 2 . However, for a nanoparticle, as opposed to a flat surface, the gold atoms near the nanoparticle surface are less strongly bound to the core atoms, and thus more mobile, allowing for the sulfur atoms to move in a third dimension normal to the nanoparticle surface. This movement normal to the nanoparticle surface has not previously been considered in the simulation of SAM-coated gold nanoparticles but is expected for the reasons discussed previously. 
The diffusivity of the alkanethiol chain is computed by considering the movement of the sulfur head group. By only using the displacement of the sulfur head group, our results are not affected by extraneous data, such as the movement of the alkanethiol backbone. In addition to studying the diffusivity of the sulfur atoms, we also calculate the diffusivity of the surface gold atoms that are bound to the sulfur atoms. By comparing the diffusivity of the surface gold atoms with that of the sulfur atoms, we can determine if the alkanethiolate chains are sliding over or moving with the gold surface atoms. For example, if the diffusivity of the sulfur atoms is very different from the gold atoms, then the sulfur atoms are moving over the nanoparticle surface, the SAM chains are considered mobile, and they may nucleate. When equation 3 is used, the diffusion coefficients for the sulfur head group and surface gold atoms are computed to be $8.05 \cdot 10^{-7}$ and $9.75 \cdot 10^{-8} \mathrm{~cm}^{2} / \mathrm{s}$, respectively. These diffusion coefficients are computed for a fully coated $50-\AA$ gold nanoparticle at $300 \mathrm{~K}$. The difference in diffusion coefficients is about 1 order of magnitude, indicating that the alkanethiolate SAM is relatively mobile on the nanoparticle surface. From observations of longer simulations ( $>10 \mathrm{~ns})$, with low surface coverage, there is no indication of nucleation of the SAM chains. This may be expected because of the attractive potential between the alkanethiolate chain and the gold surface, so that the chain monomers prefer to bond with as many gold atoms as possible in order to minimize the system energy. As such, we see no evidence at low surface coverage of free-standing groups of chains.

The diffusion coefficient of the sulfur head group has been computed for various system temperatures and presented in an Arrhenius plot in figure 3. There are two linear regions with slopes of -285.9 and -3477.6 , indicating two distinct phases of the alkanethiolate SAM in the temperature ranges of 100 to $500 \mathrm{~K}$ and 500 to $800 \mathrm{~K}$, respectively.

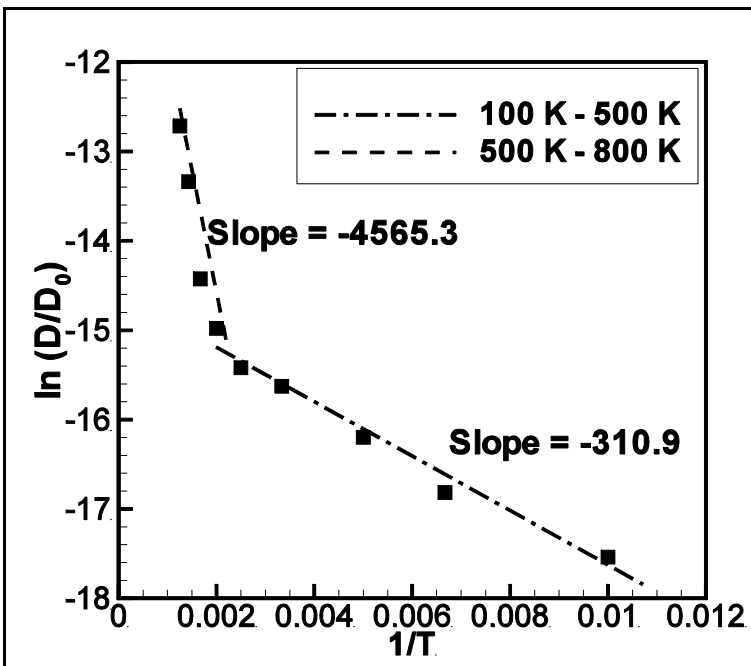

a)

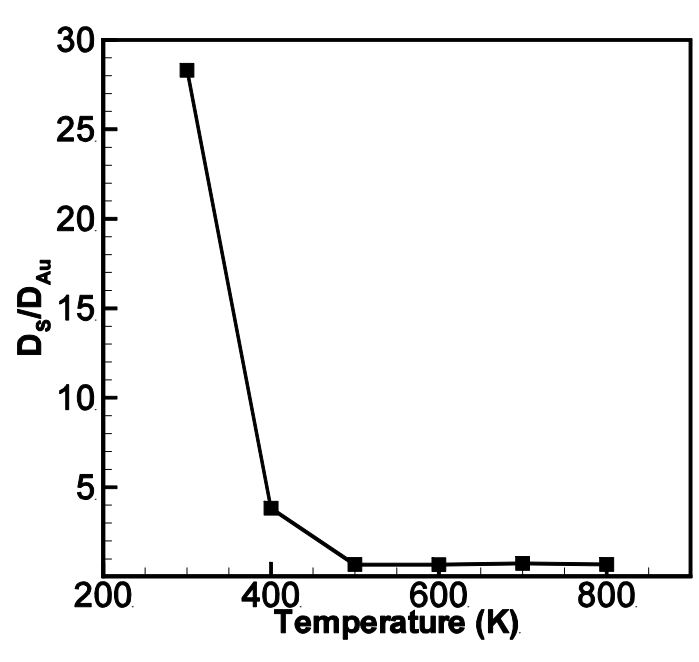

b)

Figure 3. (a) Arrhenius plot of diffusivity for sulfur atoms. The activation energy for SAM mobility is estimated by the slope of $\ln (D)$ vs. $1 / T$ between 100 and $800 \mathrm{~K}$. (b) Ratio of sulfur diffusivity to gold diffusivity in the alkanethiol-coated gold nanoparticle material system. 
The activation energy for the low- and high-temperature regions is 2.4 and $29.0 \mathrm{~kJ} / \mathrm{mol}$, respectively. This is the expected behavior for heterodiffusion, where one type of atom diffuses on another $(50,51)$. In the low-temperature regime, the observed activation energy corresponds to the alkanethiol chains hopping between adsorption sites. This process is a measure of diffusion. For flat gold surfaces, particularly the Au (111) surface, the adsorption binding energy surface has been computed using atomistic simulations $(17,52)$. These atomistic computations have determined that the difference between the minimum binding energy (face-centered cubic) site and the maximum binding energy (atop) site is between 25.1 and $16.9 \mathrm{~kJ} / \mathrm{mol}$. The diffusion barrier is the energy required for an adsorbate atom to move to the next adsorption site. In Zhang et al. (17), the authors have calculated the binding energy at a bridge site that would provide a possible diffusion path between adsorption sites with a diffusion barrier of $12.2 \mathrm{~kJ} / \mathrm{mol}$. Each of these published results are for flat surfaces and report a somewhat higher activation energy than the computed activation energy from the MD simulations for the low-temperature regime. One possible explanation for the discrepancy in the calculated value is that for a curved nanoparticle surface, there may be lower energy and irregular diffusion paths available for the alkanethiolate chains to diffuse along. More likely, however, is that all the observed activation energies are small, and differences may be associated with the accuracy of the interatomic potential. Nevertheless, the ratio of sulfur to gold diffusivity does indicate a significant difference between a low-temperature diffusion process and that occurring at a higher temperature.

Typically, as the temperature rises the dominant mechanism for diffusion shifts from adatoms to vacancies. However, what is occurring here is that the diffusion of the alkanethiolate chain is dominated by dissolution into the gold nanoparticle, resulting in a more highly activated process. In both instances, the activation energy required for diffusion is increased, but in the former process, the change in activation energy is observed to be a factor of two or more $(50)$. In this case, the change is about 1 order of magnitude. The driving force for dissolution will be discussed in the next section. We notice, however, in figure $3 \mathrm{~b}$, that the diffusivity of the gold atoms is more than an order of magnitude lower than the sulfur head group atoms at low temperatures, below $500 \mathrm{~K}$. This difference suggests that the alkanethiol chains are moving freely over the gold nanoparticle surface, but at around $500 \mathrm{~K}$ this is no longer the case, and the diffusivity of the sulfur atoms closely tracks that of the gold nanoparticle atoms. Above $500 \mathrm{~K}$, the diffusivity of the gold and sulfur atoms is similar, supporting the observation that mixing of the two materials has occurred. 


\section{Radial Pressure Distribution}

The radial pressure distribution is used in this work to quantify the effect of the adsorbed alkanethiolate SAM on the gold nanoparticle. In very small droplets, the internal pressure can be much larger than the surrounding environment. One method of evaluating the radial pressure distribution is to use the normal component of the Irving-Kirkwood (IK) pressure tensor (53). The IK pressure tensor comprises two terms, corresponding to a kinetic $P_{K}(r, T)$ and a configurational $P_{U}(r, u)$ contribution as shown in equation 4.

$$
P_{N}(r)=P_{K}(r, T)+P_{U}(r, u) \cdot
$$

The kinetic pressure term is a function of temperature and the computed radial density distribution. The configurational term is computed from the interactions, $u$, between pairs of particles. For each shell of radius $r$, the forces between particles whose line of interaction intersects the shell are considered when computing the normal pressure component. The normal pressure at the surface of a nanoparticle or droplet is typically positive, indicating a compressive surface tension. The radial pressure distribution for an uncoated 50- $\AA$ gold nanoparticle as a function of temperature is plotted in figure 4 .

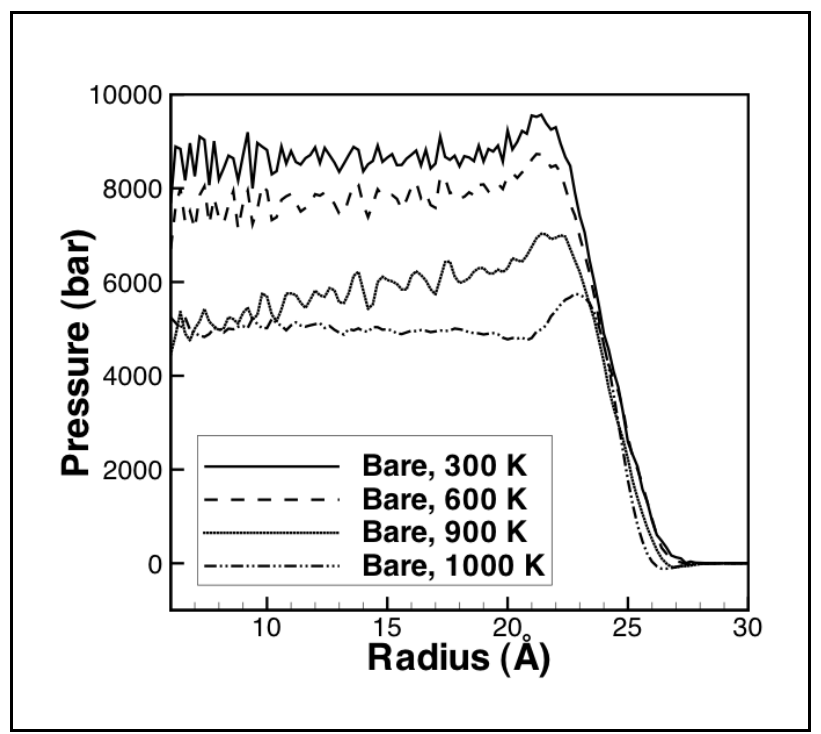

Figure 4. Radial pressure distribution for 50- $\AA$ gold nanoparticle at various temperatures.

With the gold nanoparticle melting temperature between 900 and $1000 \mathrm{~K}$, as discussed in the pair correlation function results, the "Bare, $1000 \mathrm{~K}$ " curve is for liquid gold and the other curves are for a solid gold nanoparticle. The radial pressure profile below $900 \mathrm{~K}$ shows oscillations since the particle is in the solid phase. On the other hand, the oscillation of the pressure profile at 
$1000 \mathrm{~K}$ disappears because of greater atom mobility and indicates a phase transition in agreement with the pair correlation results previously discussed. As the temperature of the nanoparticle increases, the surface pressure decreases. This decrease of pressure with increasing temperature is expected because the reported surface tension data decreases with increasing temperature. Also, from an analysis of the terms in the IK pressure tensor (equation 4), it is apparent that if the density remains relatively constant that the kinetic pressure term will increase with increasing temperature. This means that the configurational term is decreasing at an even greater rate. The magnitude of the configurational term decreases when the interactions between the gold atoms decrease or are attractive. Because the configurational pressure term is negative, the interactions between the atoms are primarily attractive. The attractive potential is due to the fact that the embedding term in the EAM potential is greater in magnitude than the core repulsive term. This occurs when the gold-gold bonds are stretched, as occurs during heating. Additionally, if the average coordination number of the gold atoms was to increase with increasing temperature, as is observed in silicon (54), then the electron density would increase. This increase in electron density causes the attractive embedding term to increase while the repulsive term, that is evaluated on a pair-wise basis, would not change.

From a dimensional analysis of the Young-Laplace (55) theorem shown as equation 5, this expected result is confirmed.

$$
\Delta P=\frac{2 \gamma}{r}
$$

In equation 5, $r$ is the radius of the gold nanoparticle, and $\Delta P$ is the difference in pressure between the interior and exterior of the droplet, i.e., $P_{\text {inside }}-P_{\text {outside }}$. Experimentally determined and published values for gold surface tension are wide ranging and available from a limited number of sources. For the solid-gas interface, the surface tension, $\gamma_{\mathrm{sg}}$, is given as $1400 \mathrm{~mJ} \cdot \mathrm{m}^{-2}$, and for the liquid-gas interface, $\gamma_{\mathrm{lg}}$ is $1128 \mathrm{~mJ} \cdot \mathrm{m}^{-2}(55)$. For a vacuum interface, $\gamma_{l_{\mathrm{v}}}$ is about $742 \mathrm{~mJ} \cdot \mathrm{m}^{-2}$ at the melting point of gold (56). It could be expected that the difference in measured surface tension between the liquid-gas and liquid-vacuum would be minimal. From the two published sources this is not the case, and so for comparison purposes we must consider these values as representative of the expected value. Evaluation of equation 5 for our $25-\AA$ radius particle and the published surface tension data for a vacuum interface result in a predicted internal pressure of $\sim 6000 \mathrm{bar}$. This analytic result is close to that obtained from the MD simulation at $1000 \mathrm{~K}$ shown in figure 4 . With the wide range of available data for comparison it can only be said that the predicted surface pressure and the simulated pressure are in reasonable agreement.

One can use the pressure profile from the MD simulation to directly compute the surface tension using the approach outlined by Rowlinson and Widom (57) and presented in equation 6. 


$$
\gamma_{i}^{3}=-\frac{1}{8}\left(p_{r_{2}}-p_{r_{1}}\right)^{2} \int_{r_{1}}^{r_{2}} r^{3} \frac{d p_{N}(r)}{d r} d r
$$

where $p_{r 2}$ and $p_{r 1}$ are the computed pressure at the maximum and minimum model radii, respectively, and $d p_{N}(r) / d r$ is the gradient of the normal pressure at radius $r$. When this model is used, the surface tension values calculated for the bare 50 - $\AA$ gold nanoparticle at 900 and $1000 \mathrm{~K}$ are 751 and $617 \mathrm{mN} / \mathrm{m}$, respectively. These temperatures bound the melting temperature of the gold nanoparticle, and the surface tension results bound the published data of $742 \mathrm{mN} / \mathrm{m}$.

The combination of the results from the Young-Laplace and Rowlinson-Widom equations shows that the internal pressure computations for the uncoated gold nanoparticle are reasonable and in the range expected. Unfortunately, there is no data available for comparison when considering the alkanethiolate-coated gold nanoparticle surface.

From past efforts (23), it is expected that a surface coating will modify the nanoparticle internal pressure, through the surface tension, of the gold nanoparticle. This possibility is considered here by comparing the radial pressure distribution for gold nanoparticles with varying degrees of surface coatings. The systems considered include a bare gold nanoparticle, a gold nanoparticle with only sulfur atoms adsorbed onto the surface, and a gold nanoparticle with alkanethiolate chains adsorbed on the surface, but with varying interaction potentials. For bare and coated gold nanoparticles with weak SAM chain-chain interactions, the internal pressure is positive, meaning a positive surface tension. This result is expected, and the trend is also reasonably expected from past research involving hydrogen-passivated silicon nanoparticles (23). As observed in figure 5, the pressure trends lower and becomes negative on the interior of the fully coated nanoparticle as the chain-chain and chain-gold interaction potential parameter values are increased to the published values, indicated by "Full Chain, 100\% Cov., $300 \mathrm{~K}$, full pot."

As a first interpretation of this negative pressure result, one could consider the volume integral of the internal pressure distribution. Since the volume integral of the pressure distribution is negative, it indicates a mechanically unstable system, or a nanoparticle under tension. As seen in figure 5, the negative pressure is observed in the interior of the nanoparticle when full chainchain and chain-gold interactions are considered. This negative pressure result indicates that the gold nanoparticle is experiencing a tensile stress and will change its configuration over time or with increases in temperature in order to accommodate the positive stress or negative pressure. The chain-chain interactions apparently have a strong influence on the surface tension and therefore the internal pressure of the nanoparticle. The strength of the interaction potential that will flip the internal pressure from positive and mechanically stable to negative and mechanically unstable is still under investigation but is between $10 \%$ and $100 \%$ of the published potentials used for the alkanethiolate SAM interaction potential. Other factors that may affect the surface tension are the surface area per adsorbed alkanethiol chain, and temperature. In figure 6 , the 


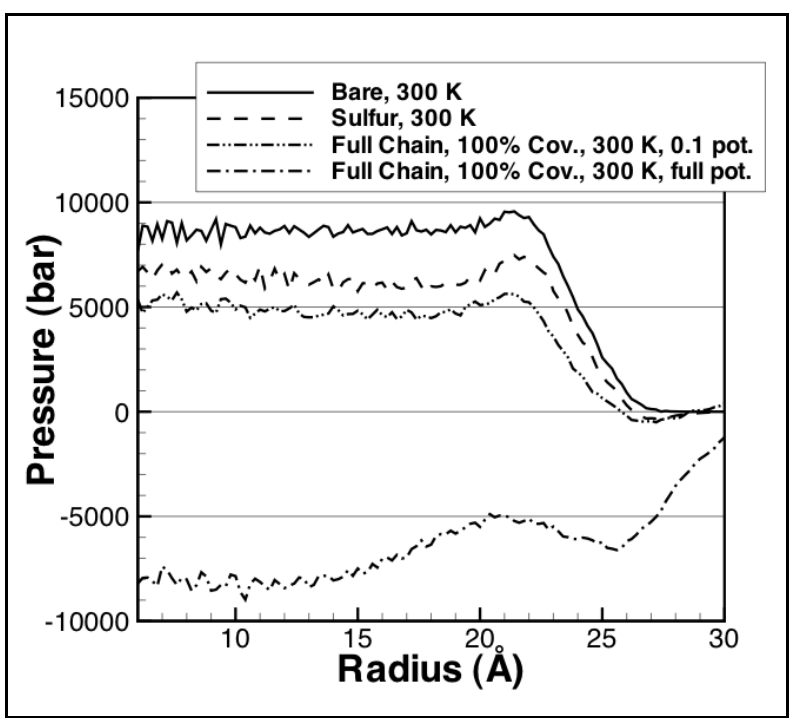

Figure 5. Radial pressure distribution for a 50-Å gold nanoparticle with alkanethiolate SAMs with differing interaction coefficients.

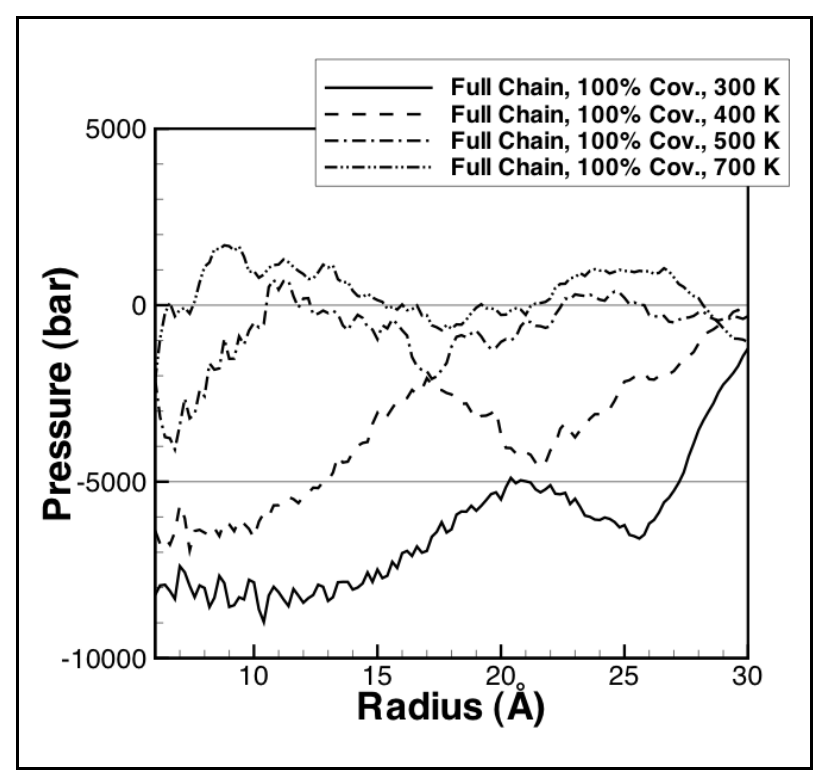

Figure 6. Radial pressure distribution for a fully coated gold nanoparticle for range of temperatures with high binding energy $(184.1 \mathrm{~kJ} / \mathrm{mol})$.

radial pressure profile is plotted for a fully coated gold nanoparticle with a sulfur-gold binding energy of $184.1 \mathrm{~kJ} / \mathrm{mol}$. Notice that as the temperature increases, the pressure that is primarily negative at $300 \mathrm{~K}$ becomes positive at $700 \mathrm{~K}$. As the system is held at a temperature above the melting temperature of the solution, the pressure continues to increase and will most likely equilibrate to an average pressure above 0. 
A negative surface tension resulting in a tensile stress inside the nanoparticle is not stable for a liquid nanoparticle and will result in deformation. In a solid nanoparticle, the tensile stress will exist for a time before creep (58) or an increase in temperature will lower the yield strength allowing the nanoparticle to yield and flow. For these reasons, the solid gold nanoparticle with an alkanethiolate coating is metastable at low temperatures but will stabilize at higher temperatures by changing shape. In this particular case, we observed that the alkylthiol chains dissolve into the particle, as discussed in the section on diffusion. This result has not been previously observed in computer simulations because the gold nanoparticle was assumed to maintain its shape, with the interaction of the alkanethiolate SAM (18) constrained to the surface. Using the lower binding energy $(126 \mathrm{~kJ} / \mathrm{mol})$, we initially observe desorption of alkanethiolate chains from the nanoparticle surface as the temperature is increased, until the temperature is above $600 \mathrm{~K}$ at which time the remaining alkanethiolate chains dissolve into the nanoparticle.

In figure 7, the internal pressure of the alkanethiol-coated gold nanoparticle is plotted from simulation data collected with a sulfur-gold binding energy of $126 \mathrm{~kJ} / \mathrm{mol}$.

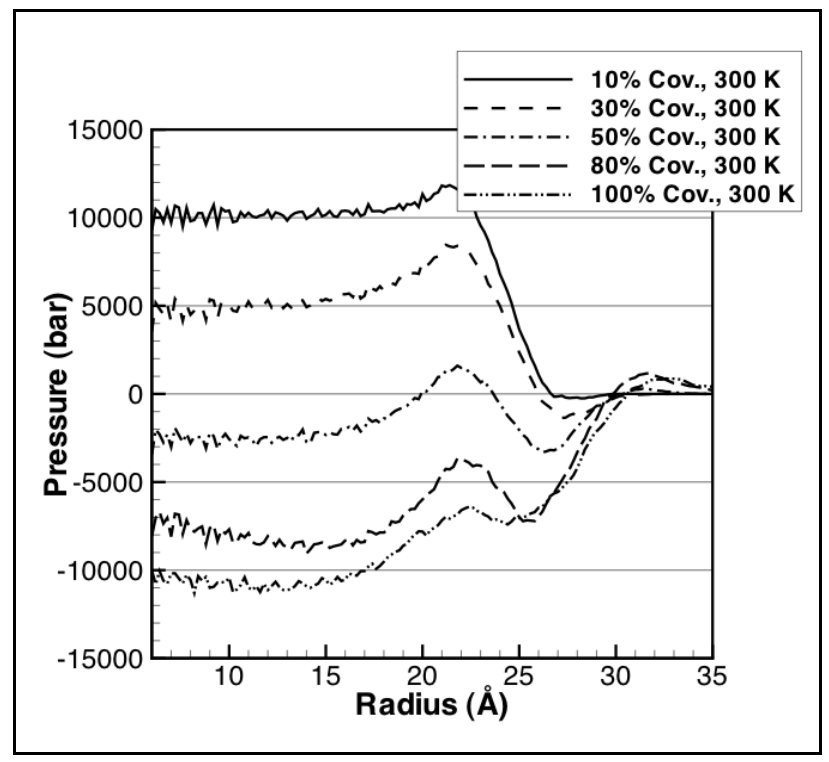

Figure 7. Radial pressure distribution for alkanethiolcoated 50-Å gold nanoparticle with $126 \mathrm{~kJ} / \mathrm{mol}$ sulfur-gold binding energy.

Notice in figure 7 that the internal pressure of the fully coated nanoparticle with the $126 \mathrm{~kJ} / \mathrm{mol}$ sulfur binding energy is very similar to the internal pressure of the fully coated gold nanoparticle in figure 5, where the binding energy of the sulfur-gold bond is $184 \mathrm{~kJ} / \mathrm{mol}$. Recall that only the sulfur-gold binding energy has been changed in figure 7 , whereas in figure 5 , the " 0.1 pot." curve represents data collected from simulations where the sulfur-gold and the chain-chain interactions have both been modified. By comparing these results, we can conclude that the chain-chain interactions have a greater effect on internal pressure than the sulfur-gold binding energy. This is an important result because although the sulfur-gold binding energy has some effect on the 
structure of the gold nanoparticle and diffusivity, it is not the primary factor in determining stability of the nanoparticle. Additionally, the effect of chain length on the radial pressure distribution is also considered. After computing the radial pressure distribution for alkanethiol chain lengths of 3 and 18 carbon atoms, we have found that the shorter chains result in an internal pressure about 5000 bar higher than the previously considered 9 carbon atom chains. Alternatively, the longer chains don't appear to have a greater effect on the internal pressure of the nanoparticle than the nine carbon atom chains studied here. This leads to the conclusion that for the number of monomers considered, a longer chain has little effect on the stability of the nanoparticle, whereas a shorter chain will lower the total chain-chain interaction sufficiently to affect the nanoparticle stability.

\section{Radial Density Distribution}

From the computed results up to this point, we have determined that the 50- $\AA$ uncoated gold nanoparticle has a melting temperature between 900 and $1000 \mathrm{~K}$ (pair correlation function), the SAM chains are mobile on the gold surface (diffusion coefficient), and there are two phases for the adsorbed alkanethiolate SAM between 100 and $800 \mathrm{~K}$. In this section, the radial density results are analyzed. The radial density is computed as part of the radial pressure calculation discussed previously. For bare nanoparticles, the radial density distribution for the gold atoms will change abruptly at the nanoparticle surface from the core density to 0 . On the other hand, for coated nanoparticles, if the surface atoms move radially outward due to the presence of the alkanethiolate chains penetrating into the particle, the slope of the radial density distribution will be less steep. This phenomenon of the SAM chains penetrating into the nanoparticle surface is not a previously predicted result because past efforts to simulate alkanethiolate-coated gold nanoparticles have assumed that the gold atoms do not move as a result of the interactions with the SAM $(18,40)$. These previous results have either assumed a flat surface or a nanocrystallite with immobile atoms. Modeling the gold atoms using the EAM potential provides for an investigation of whether or not the gold atoms are affected by the SAM. When the radial density is analyzed, shown in figure 8 , it is apparent that the atoms near the surface of the gold nanoparticle are less densely packed than those in the nanoparticle core. This decrease in gold atomic density suggests that near the nanoparticle surface, the gold atoms are mixing with the alkanethiolate molecules.

The results in figure 8 show the expectedly steep slope for the radial density distribution of the uncoated gold nanoparticle. The slope is not vertical because the nanoparticle is nonspherical but rather has large facets (9). The slope of the radial density plot for the coated gold nanoparticle is less steep than the uncoated nanoparticle. The slope decreases monotonically as more SAM chains are adsorbed onto the gold surface. This decrease in slope indicates that the 


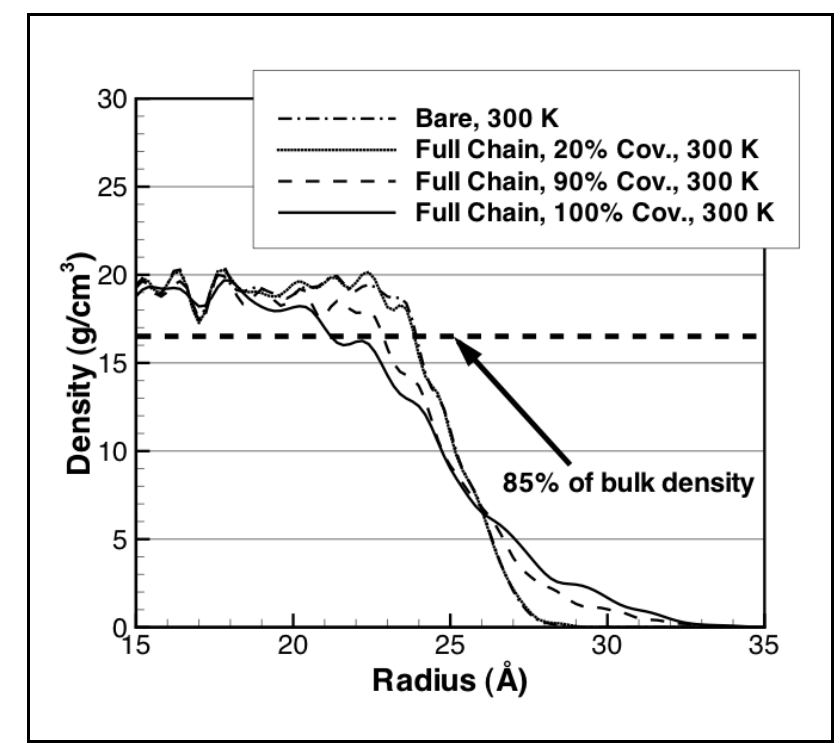

Figure 8. Radial density of gold in alkanethiolate SAMcoated gold nanoparticle for various surface coverage amounts.

atoms near the surface are more diffuse, resulting in the lower measured density of gold. We have labeled the depth to which the gold core is affected as the surface corrugation.

In order to quantify the surface corrugation, we have a corrugation factor, $C$, defined by equation 7 .

$$
C=\frac{r\left(\rho=0.85 \rho_{\text {bulk }}\right)_{\text {bare }}-\mathrm{r}\left(\rho=0.85 \rho_{\text {bulk }}\right)_{\text {coated }}}{r\left(\rho=0.85 \rho_{\text {bulk }}\right)_{\text {bare }}} .
$$

In equation $7, r\left(\rho=0.85 \rho_{\text {bulk }}\right)$ is the radial position where the computed gold density is $85 \%$ of the density of bulk gold, and the subscripts bare and coated refer to the uncoated and alkanethiolate SAM-coated gold nanoparticles, respectively. This definition of the corrugation factor will produce a value of 0.0 for the bare gold nanoparticle and a maximum of 1.0 for a gold nanoparticle with impurities or a dissolved solute. For coated gold nanoparticles with various percentages of surface coverage, the corrugation factor has been computed and is listed in table 1. 
Table 1. Computed corrugation factors for alkanethiolate SAM-coated gold nanoparticles.

\begin{tabular}{|c|c|c|c|}
\hline Coverage & $\mathbf{C}_{\mathbf{1 8 4} \mathbf{~ k J} / \mathbf{m o l}}$ & $\mathbf{C}_{\mathbf{1 2 6} \mathbf{k J} / \mathbf{m o l}}$ & $\mathbf{C}_{\text {weak chain }}{ }^{\mathbf{a}}$ \\
\hline Bare & 0.000 & 0.000 & NA \\
\hline $10 \%$ & 0.004 & 0.003 & NA \\
\hline $70 \%$ & 0.012 & 0.018 & NA \\
\hline $80 \%$ & 0.018 & 0.013 & NA \\
\hline $90 \%$ & 0.043 & 0.020 & NA \\
\hline $100 \%$ & 0.107 & 0.081 & 0.019 \\
\hline
\end{tabular}

${ }^{a}$ The gold-sulfur binding energy is $184 \mathrm{~kJ} / \mathrm{mol}$, but all chain-chain interactions are at $10 \%$.

The results presented in table 1 show that the corrugation factor remains small, indicating little change in the nanoparticle surface, for SAM coverage below $90 \%$. Once the coverage reaches $90 \%$, the corrugation factor increases 2.5 times over the value at $80 \%$ and increases by another factor of 2.5 times between $90 \%$ and $100 \%$. With a corrugation value of 0.107 in column $\mathrm{C}_{184 \mathrm{~kJ} / \mathrm{mol}}$, at full surface coverage the core of the gold nanoparticle is only about $89 \%$ of its original size. This result has two implications: (1) the surface area of the gold nanoparticle has increased because the surface is now wrinkled and no longer smooth, and (2) the SAM chains are able to form more bonds to the increased number of exposed gold atoms, potentially increasing the binding energy of the SAM to the gold nanoparticle. The results in table 1 indicate an exponential dependence of the corrugation factor on the amount of surface coverage.

In the column for the lower sulfur-gold binding energy, namely $\mathrm{C}_{126 \mathrm{~kJ} / \mathrm{mol}}$, the corrugation is less pronounced indicating a lower surface effect from the adsorbed SAM. The difference in magnitude of corrugation between the high and low binding energies is relatively small, about $25 \%$. This small change in corrugation from a $33 \%$ change in gold-sulfur binding energy may indicate that the gold-sulfur binding energy is not the most important factor in determining surface corrugation. In the final column of table 1 , the gold-sulfur binding energy is $184 \mathrm{~kJ} / \mathrm{mol}$, but all chain-chain interactions have been lowered to $10 \%$ of the previous magnitudes. Only the $100 \%$ surface coverage data is computed, but by comparing this result with the other columns it is apparent that the chain-chain interaction has an appreciable effect on the surface corrugation. The $90 \%$ drop in chain-chain interactions is accompanied by an $83 \%$ drop in corrugation. This is proportional to the change in corrugation observed by lowering the sulfur-gold binding energy alone.

There is experimental support for corrugation of the gold surface from the adsorption of an alkanethiol SAM (59). In the experimental measurements, it is assumed that an interior gold atom is pulled onto the surface to become an adatom. In this case, the alkanethiol then bonds atop this gold adatom, and the gold surface is thus corrugated. Another consequence of this adatom is that the alkanethiol is more mobile and could be construed as a lower total sulfur-togold surface binding energy than if the sulfur atom were in the vicinity of many gold atoms. A 
rough estimate of the measured corrugation in this case would be the gold-gold bond distance divided by the nanoparticle radius, resulting in $\mathrm{C}_{\exp }=0.116$, a result similar to the values in table 1 for a $100 \%$ coated surface.

\section{Phase Behavior and SAM Solubility}

The computed potential energy (PE) of the alkanethiolate SAM-coated gold nanoparticle system is used to determine the critical temperature at which mixing and phase change will occur. The computed slope of the PE vs. temperature curve from the MD simulation results is used to determine the heat capacity of the system.

In figure 9, the system PE is plotted vs. temperature for a 50- $\AA$ gold nanoparticle coated with increasing densities of the alkanethiolate SAM. For the bare nanoparticle, there is a discontinuity around $980 \mathrm{~K}$, which corresponds to a phase change. The SAM-coated nanoparticle demonstrates a very different behavior. Initially, for small numbers of adsorbed alkanethiolate chains, the melting temperature of the gold nanoparticle decreases and does not occur at a unique temperature but rather over a range of temperatures. This behavior is expected for a mixture of two materials (55). The appearance of the SAM on the particle surface decreases the surface tension and internal pressure. As the coverage percentage is increased further, another behavior is observed. At higher surface coverages, above $70 \%$, the PE of the system begins to decrease as the temperature is increased, and then after a small temperature range the PE resumes its linear increase. This behavior is explained by the mixing that occurs between the alkanethiolate chains and the gold atoms. The mixing of the SAM chains with the gold atoms is possible because of the lower binding energy of surface Au atoms. The lower binding energy of the surface atoms allows these atoms to become mobile at a lower temperature than the interior gold atoms (12). The surface atoms begin to mix with the alkanethiol chains, and as the chains penetrate deeper into the nanoparticle, they are able to interact with an ever increasing number of gold atoms. Once complete mixing has occurred, the PE resumes increasing monotonically. 


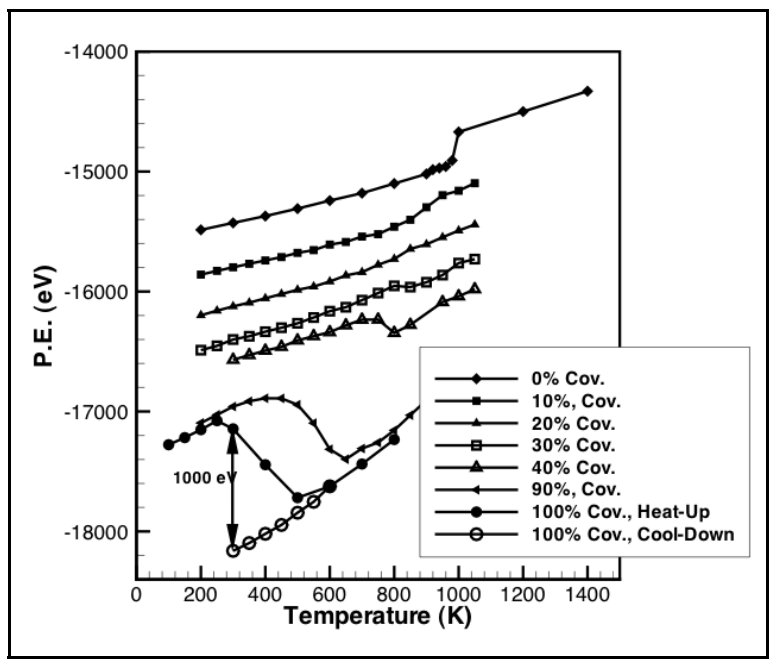

Figure 9. PE vs. temperature for the alkanethiolate SAM-coated gold nanoparticle with various amount of surface coverage and the 184 $\mathrm{kJ} / \mathrm{mol}$ sulfur binding energy.

As we found with the internal pressure in figure 5, the structure of the SAM-coated gold nanoparticle is metastable. The increase of both the surface coverage and the temperature aids this particle in transforming into a mixed structure, figure 10. This result has not been previously predicted, as it was previously assumed that the SAM chains would desorb at these temperatures, as occurs on flat surfaces (17). If the SAM were to desorb, it is expected that the structure of the underlying gold nanoparticle would not be affected. This is reflected in the pair correlation data discussed earlier that shows that the gold nanoparticle with SAM coating is crystalline at a higher temperature with the lower $126 \mathrm{~kJ} / \mathrm{mol}$ sulfur binding energy than with the $184 \mathrm{~kJ} / \mathrm{mol}$ binding energy.

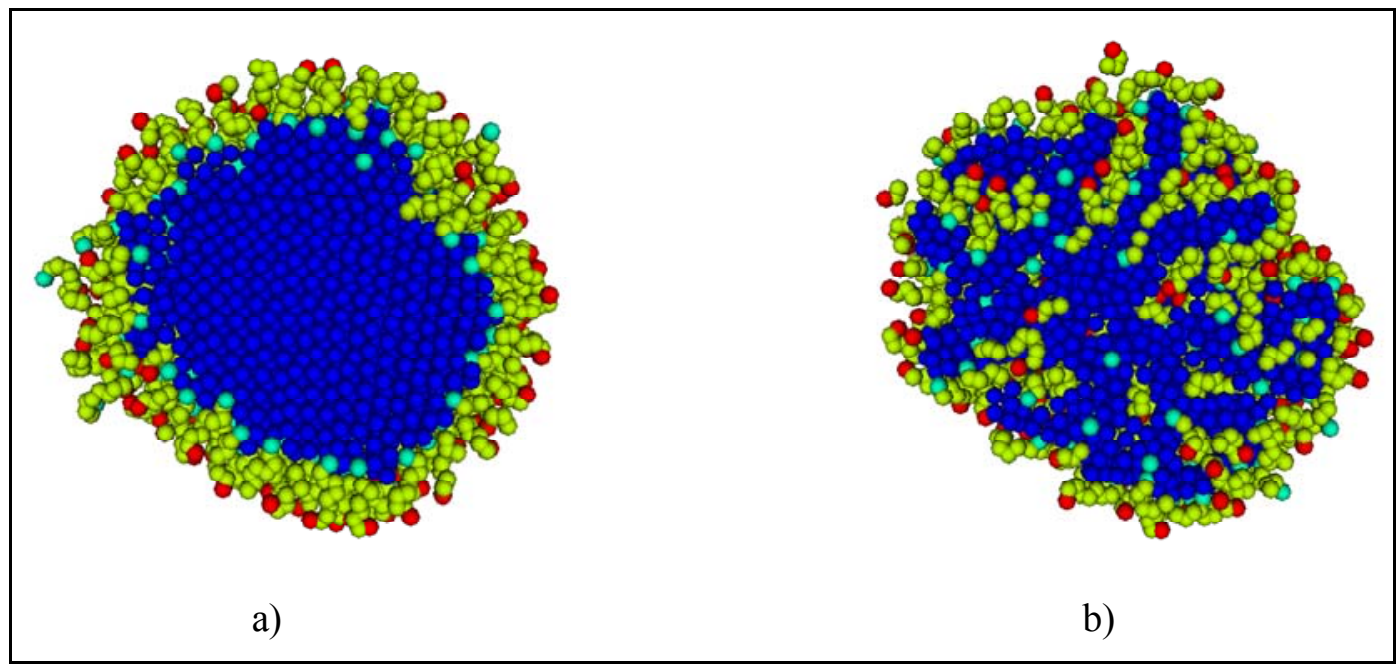

Figure 10. Cross section of $100 \%$ coated gold nanoparticle at (a) $300 \mathrm{~K}$ and (b) $600 \mathrm{~K}$, showing the dissolution of the alkanethiol chains at high temperatures. (The blue spheres represent gold atoms, light blue are sulfur, yellow are $\mathrm{CH}_{2}$, and red are $\mathrm{CH}_{3}$.) 
The PE for the $126 \mathrm{~kJ} / \mathrm{mol}$ sulfur binding energy simulation is plotted in figure 11a. During these simulations, as opposed to the $184 \mathrm{~kJ} / \mathrm{mol}$ binding energy simulations, we observed some desorption of alkanethiol chains from the gold surface. This desorption process is irreversible because of the low density of desorbed chains in the simulation volume, a realistic assumption for coated nanoparticles in a vacuum or near vacuum. In figure $11 \mathrm{~b}$, it is interesting to note that the temperature at which the decrease in PE occurs for the $126 \mathrm{~kJ} / \mathrm{mol}$ sulfur binding energy is higher than the $184 \mathrm{~kJ} / \mathrm{mol}$ binding energy simulations. This result confirms the observation that when considered with a lower-sulfur binding energy, the alkanethiol chains have a lower propensity to dissolve into the gold nanoparticle and a higher probability of desorbing from the gold surface.

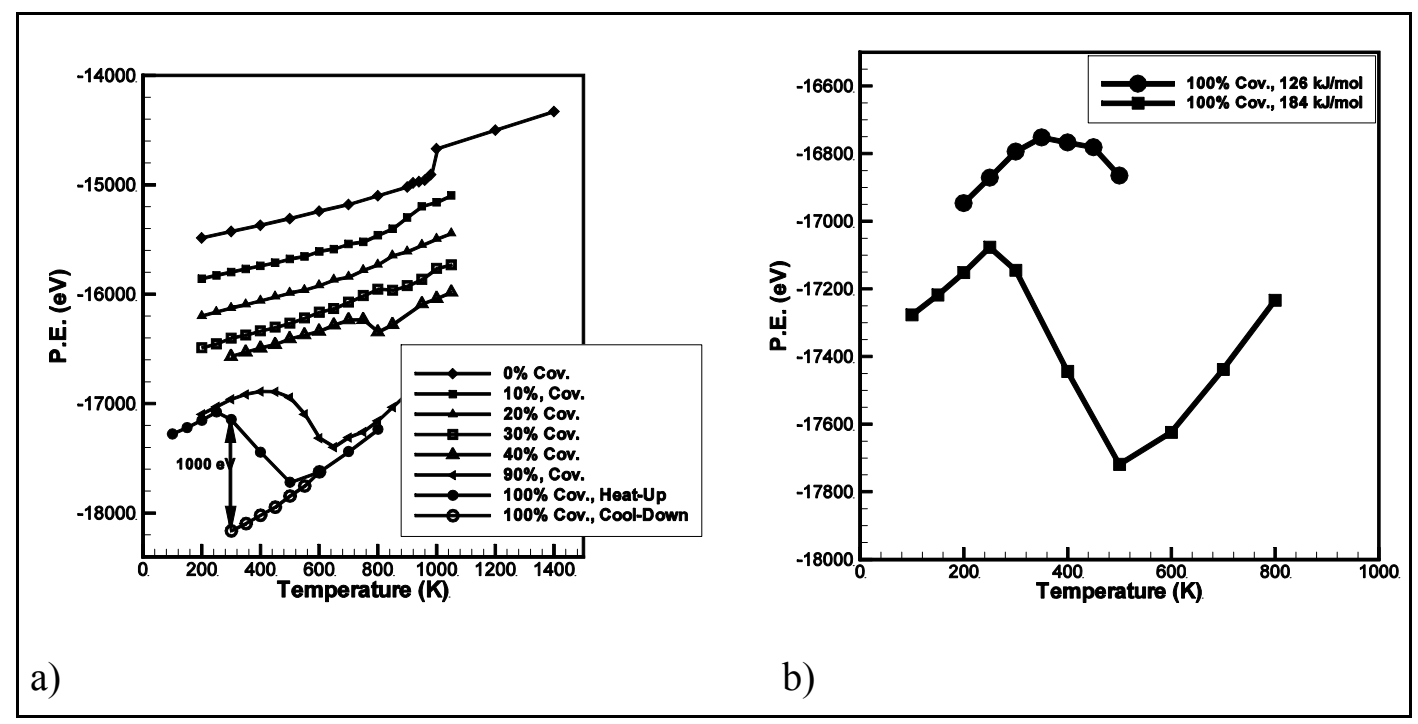

Figure 11. (a) PE vs. temperature for the alkanethiolate SAM-coated gold nanoparticle with various amounts of surface coverage and $126 \mathrm{~kJ} / \mathrm{mol}$ binding energy. (b) Comparison of PE vs. temperature for fully coated nanoparticle with 184 and $126 \mathrm{~kJ} / \mathrm{mol}$ binding energy.

The constant volume heat capacity, $C_{V}$, of the nanoparticle system is computed directly from the slope of the PE vs. temperature curve. The portion of the PE vs. temperature curve used to determine the heat capacity is that below the melting temperature or prior to mixing. For the nanoparticles studied in figure 9 , the specific heat capacity, $c_{V}$, is computed and plotted in figure 12 for increasing amounts of surface coverage. In figure 12 , the computed heat capacity of the system per unit mass is increasing with increasing surface coverage. This result is reasonable since gold has a relatively low specific heat compared with the alkanethiolate chains. As more chains are added to the nanoparticle surface, the fraction of the total system mass that they represent increases, resulting in the increase in the system heat capacity. 


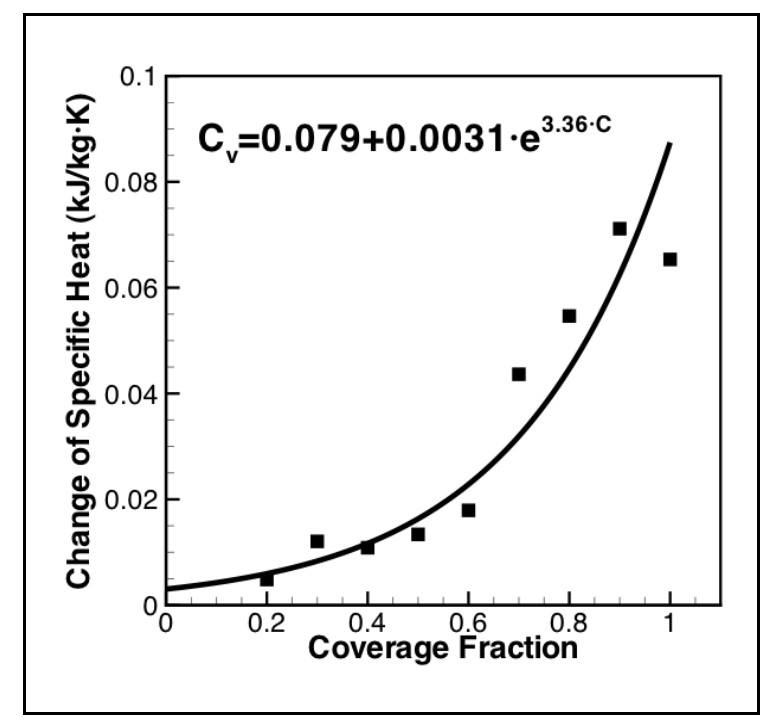

Figure 12. Plot of specific heat vs. surface coverage for alkanethiolate SAM-coated gold nanoparticle showing exponential relationship between specific heat and surface coverage.

In addition to the heat capacity, the enthalpy of solution, $\mathrm{H}_{\text {sol }}$, can be computed from the PE data. The enthalpy of solution is computed as the difference in system internal energy between the undissolved SAM chain system prior to heating and the internal energy of the same system after cooling down to the same temperature. The PE of the system decreases as a result of the mixing that occurs in the fully coated gold nanoparticle model during an increase in temperature from 300 to $600 \mathrm{~K}$. This mixing may potentially occur completely at $300 \mathrm{~K}$ if it were possible to simulate long times ( $>10 \mathrm{~ns}$ ) in the MD simulations performed here. On the other hand, it is also possible that the activation energy needed for this transition to occur may be too high to complete the transition at $300 \mathrm{~K}$. The measured change in system PE is $1000 \mathrm{eV}$, which is the enthalpy of solution (55). Denoting this result per unit mass of the fully coated gold nanoparticle results in an enthalpy of solution of $100 \mathrm{~kJ} / \mathrm{kg}$. These results show that the SAM has a measurable effect on not only the nanoparticle surface, but also the entire system.

\section{SAM Phases}

Determining the phase diagram of the SAM chains on a gold surface is of fundamental interest. There has been extensive effort to understand the various phases of the alkanethiolate SAM chain on flat gold surfaces (60) that have found four distinct phases. The four phases are a striped phase at low surface coverage, where the chains lie flat along the gold surface, 
intermediate structures for higher surface coverage, where some chains are partially standing, and others are lying flat. At high surface coverage, the alkanethiolate chains are either in a highly structured $\mathrm{c}(4 \times 2)$ phase $(7)$ at low temperatures or a more random liquid phase at higher temperatures. In this work, as in previous work involving flat surfaces, we have investigated surface coverage and temperature as determining factors for the phase of the SAM.

It is expected that the binding energy of an alkanethiolate chain to the gold surface is dependent on whether the chain is lying along the surface of the nanoparticle (physisorption for longer chains or chemisorption for shorter chains) or standing radially outward with only the head group sulfur atom interacting with the gold surface (chemisorption) (45). The orientation of the chain is determined by measuring the tilt angle of the alkanethiol chain, which is computed by comparing the radial position of the sulfur head group to the $\mathrm{CH}_{3}$ tail group. For an alkanethiol chain lying along the nanoparticle surface, the difference in radial position between the head and tail groups will be very small. When a chain is tilted upward, the radius will be at a maximum value. This radius data is then used to estimate the angle that the chains make with the nanoparticle surface and to measure the monolayer thickness (61).

In figure $13 \mathrm{~b}$, the tilt angle of the alkanethiol SAM is plotted for $10 \%$ to $100 \%$ surface coverage. At low surface coverage, the alkanethiol chains lie along the surface of the nanoparticle as indicated by the almost $90^{\circ}$ tilt angle. In between the high and low surface coverage regions, the alkanethiolate chains begin to stand radially outward from the nanoparticle surface. Above $90 \%$ surface coverage, the chains have all orientated themselves with the minimally observed tilt angle from the nanoparticle surface, and the maximum difference in radial position is observed. The tilt angle for the alkanethiolate SAM on a flat gold surface is also plotted for comparison. The steeper drop in angle and minimum tilt angle are both attributable to the geometric issues discussed next and illustrated in figure 14.

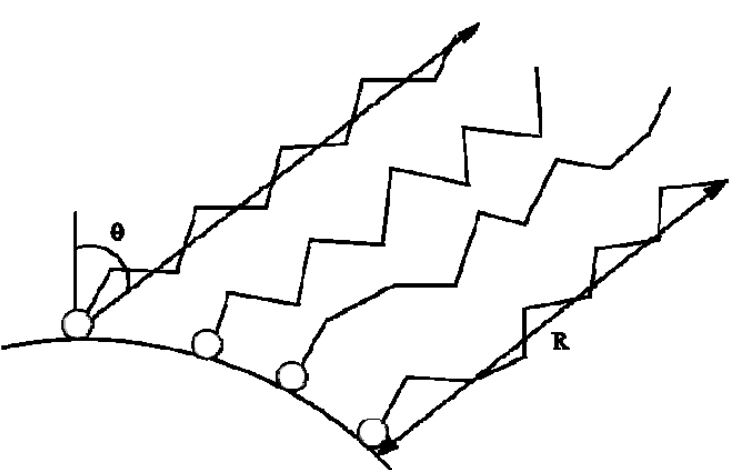

a)

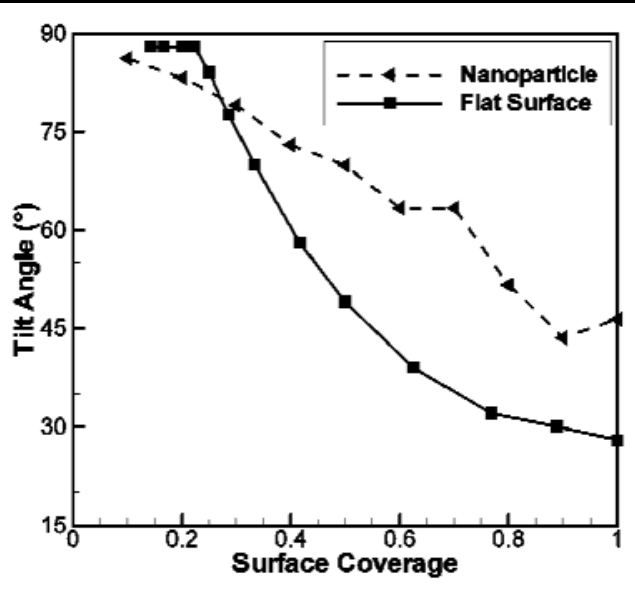

b)

Figure 13. Difference in radial position for the head and tail groups of the alkanethiolate chains at various surface coverage densities, (a) drawing and (b) tilt angle $(\theta)$. The alkanethiol radius is also labeled as the monolayer thickness. 


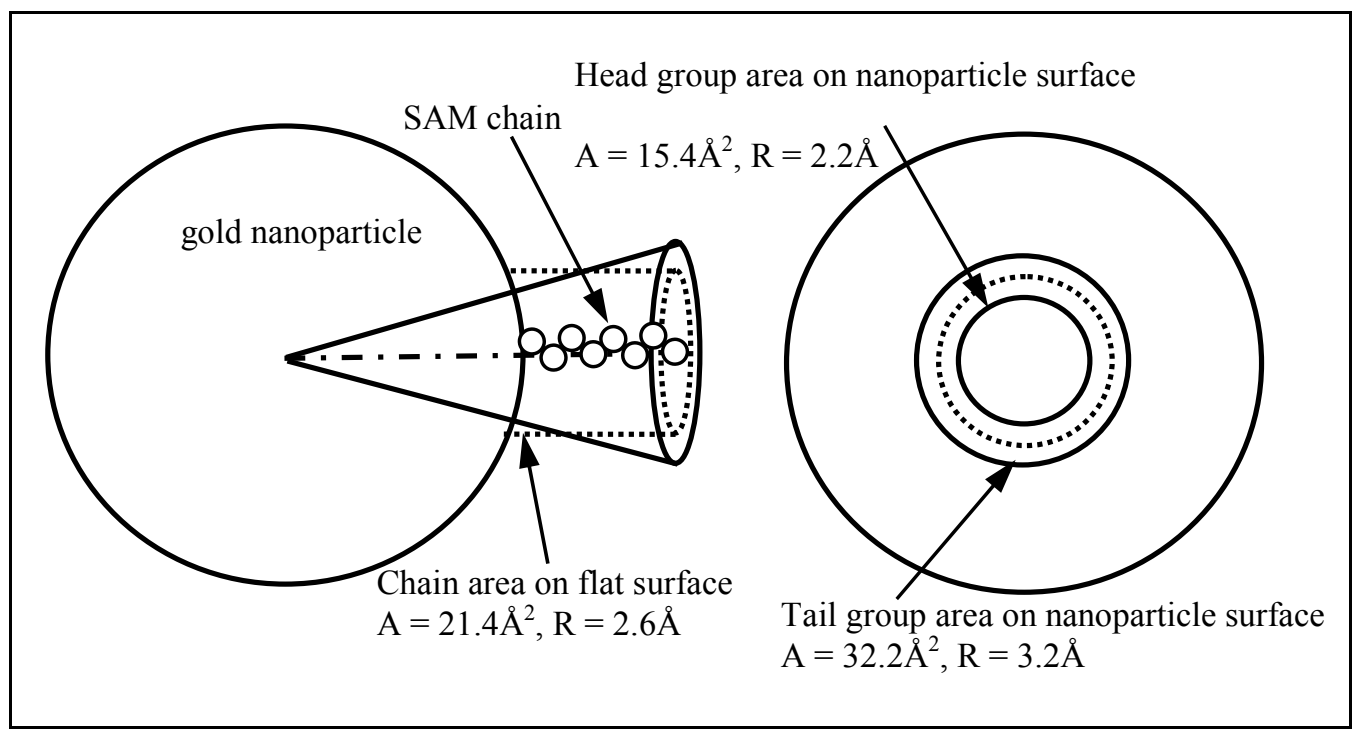

Figure 14. Drawing showing the difference in space occupied by tail molecule on curved and flat gold surfaces, assuming a chain length of nine carbon atoms.

In figure 14, a side and front view of an idealized spherical nanoparticle are illustrated with an alkanethiol chain extending radially from the nanoparticle surface. The conic section shows the volume associated with an alkanethiol chain adsorbed to the surface of a nanoparticle. The cylinder is the volume associated with an alkanethiol chain on a flat surface. Notice that the area of the cone sliced at the nanoparticle surface is smaller $\left(15.4 \AA^{2}\right)$ than the area of the cylinder $\left(21.4 \AA^{2}\right)$. This gives rise to the higher packing density on the nanoparticle surface compared to the flat gold surface. At the tail of the alkanethiol chain, for chains with nine carbon atoms as considered here, the cone has a larger cross-sectional area than the cylinder. This larger area means that the tail of an alkanethiol chain has less restricted movement than the tail of an alkanethiol chain adsorbed onto a flat surface. This greater freedom of movement results in the higher tilt angle measured in this work $\left(42^{\circ}\right)$ compared to the tilt angle measured for a flat gold surface (about $\left.30^{\circ}\right)(7,17)$.

\section{Conclusions}

The alkanthiolate SAM-coated gold nanoparticle has been shown to be mechanically and chemically metastable. The SAM that is adsorbed onto the surface begins to mix with the surface gold atoms at low temperatures because of the weaker gold-gold bonding of the surface atoms to the interior gold atoms. This process is more pronounced as the density of the SAM is increased and the gold-sulfur binding energy is increased. This is demonstrated by the corrugation numbers computed previously. For full surface coverage, the SAM chains will begin to diffuse into the gold core as the temperature rises from 300 to $500 \mathrm{~K}$. This mixing process lowers the PE of the system. The diffusion activation energy of the sulfur atoms is measured to 
be low in this temperature range. Once the temperature has increased to around $600 \mathrm{~K}$, the alkanethiolate chains are completely mixed with the gold atoms, and the activation energy for diffusion greatly increases. The PE and diffusivity results concur with this observation that a phase change in the SAM-coated gold nanoparticle system occurs around $500 \mathrm{~K}$. We have therefore concluded that the melting temperature of the gold is lowered by the adsorption of alkanethiolate SAMs on the nanoparticle surface and creates a metastable system for fully covered nanoparticles of diameters around $50 \AA$. The measured decrease in melting temperature of the nanoparticle is more pronounced as more SAM chains are adsorbed onto the nanoparticle surface.

We have also compared each of the computed results for the low, $126 \mathrm{~kJ} / \mathrm{mol}$ gold-sulfur binding energy reported in the literature to the results from the high, $184 \mathrm{~kJ} / \mathrm{mol}$ binding energy. These comparisons show that the gold-sulfur binding energy does not have a large effect on the metastability of the nanoparticle. From these results, we are able to conclude that the primary factor in determining if a nanoparticle will become metastable is the strength of the chain-chain interactions. Since experiments concur that desorption occurs in the 300 to $550 \mathrm{~K}$ temperature range, we have concluded that the $126 \mathrm{~kJ} / \mathrm{mol}$ binding energy is most likely a better estimate of the true binding energy than the $184 \mathrm{~kJ} / \mathrm{mol}$ value. Independent of the binding energy is the tilt angle, which is found to be greater for the nanoparticle geometry than for a flat surface. 


\section{References}

1. Manna, A.; Imae, T.; Aoi, K.; Okazaki, M. Gold Nanoparticles Self-Confined by Hybrid Self-Assembled Monolayers of Dendrimer and Dodecanethiol. Mol. Sim. 2003, 29 (10-11), 661-665.

2. Hong, R.; Han, G.; Fernández, J. M.; Kim, B. J.; Forbes, N. S.; Rotello, V. M. GlutathioneMediated Delivery and Release Using Monolayer Protected Nanoparticle Carriers. J. Am. Chem. Soc. 2006, 128, 1078-1079.

3. Magnusson, M. H.; Duppert, K.; Malm, J. O.; Bovin, J. O.; Samuelson, L. Size-Selected Gold Nanoparticles by Aerosol Technology. Nanostruct. Mater. 1999, 12, 45-48.

4. Chiang, C. L. Controlled Growth of Gold Nanoparticles in Aerosol-OT/Sorbitan Monooleate/Isooctane Mixed Reverse Micelles. J. Colloid Interface Sci. 2000, 230, 60-66.

5. Kim, J. U.; Cha, S. H.; Shin, K.; Jho, J. Y.; Lee, J. C. Synthesis of Gold Nanoparticles From Gold(I)-Alkanethiolate Complexes With Supramolecular Structures Through Electron Beam Irradiation in TEM. J. Am. Chem. Soc. Communications 2005, 127, 9962-9963.

6. Landman, U.; Luedtke, W. D. Small Is Different: Energetic, Structural, Thermal, and Mechanical Properties of Passivated Nanocluster Assemblies. Faraday Discuss. 2004, 125, $1-22$.

7. Schreiber, F. Structure and Growth of Self-Assembling Monolayers. Prog. Surf. Sci. 2000, $65,151-256$.

8. Greer, J. R.; Nix, W. D. Size Dependence of Mechanical Properties of Gold at the SubMicron Scale. Appl. Phys. A 2005, 80, 1625-1629.

9. Cleveland, C. L.; Landman, U.; Schaaff, T. G.; Shafigullin, M. R.; Stephens, P. W.; Whetten, R. L. Structural Evolution of Smaller Gold Nanocrystals: The Truncated-Decahedral Motif. Phys. Rev. Lett. 1997, 79 (10), 1873-1876.

10. Baskes, M. I. Modified Embedded-Atom Potentials for Cubic Materials and Impurities. Phys. Rev. B 1992, 46 (5), 2727-2742.

11. Daw, M. S.; Baskes, M. I. Embedded-Atom Method: Derivation and Application to Impurities, Surfaces, and Other Defects in Metals. Phys. Rev. B 1984, 29 (12), 6443-6453.

12. Lewis, L. J.; Jensen, P.; Barrat, J. L. Melting, Freezing, and Coalescence of Gold Nanoclusters. Phys. Rev. B 1997, 56 (4), 2248-2257. 
13. Shevade, A. V.; Zhou, J.; Zin, M. T.; Jiang, S. Phase Behaviour of Mixed Self-Assembled Monolayers of Alkanethiols on $\mathrm{Au}(111)$ : A Configurational-Bias Monte Carlo Simulation Study. Langmuir 2001, 17, 7566-7572.

14. Nuzzo, R. G.; Zegarski, B. R.; Dubois, L. H. Fundamental Studies of the Chemisorption of Organosulfur Compounds on Au(111). Implications for Molecular Self-Assembly on Gold Surfaces. J. Am. Chem. Soc. 1987, 109, 733-740.

15. Strong, L.; Whitesides, G. M. Structures of Self-Assembled Monolayer Films of Organosulfur Compounds Adsorbed on Gold Single Crystals: Electron Diffraction Studies. Langmuir 1988, 4, 546-558.

16. Rosenbaum, A. W.; Freedman, M. A.; Darling, S. B.; Popova, I.; Sibener, S. J. Surface Vibrations in Alkanethiol Self-Assembled Monolayers of Varying Chain Length. J. Chem. Phys. 2004, 120 (8), 3880-3886.

17. Zhang, L.; Goddard, W. A., III; Jiang, S. Molecular Simulation Study of the c(4x2) Superlattice Structure of Alkanethiol Self-Assembled Monolayers on Au(111). Comput. Chem. Eng. 2002, 22 (10), 1381-1385.

18. Luedtke, W. D.; Landman, U. Structure, Dynamics, and Thermodynamics of Passivated Gold Nanocrystallites and Their Assemblies. J. Phys. Chem. 1996, 100 (32), 13323-13329.

19. Jiang, S. Molecular Simulation Studies of Self-Assembled Monolayers of Alkanethiols on Au(111). Mol. Phys. 2002, 100 (14), 2261-2275.

20. Bhatia, R.; Garrison, B. J. Phase Transitions in a Methyl-Terminated Monolayer SelfAssembled on $\mathrm{Au}\{111\}$. Langmuir 1997, 13, 765-769.

21. Masens, C.; Ford, M. J.; Cortie, M. B. The Effect of Surface Symmetry on the Adsorption Energetics of $\mathrm{SCH}_{3}$ on Gold Surfaces Studied Using Density Functional Theory. Surf. Sci. 2005, 580, 19-29.

22. Hawa, T.; Zachariah, M. R. Molecular Dynamics Study of Particle-Particle Collisions Between Hydrogen-Passivated Silicon Nanoparticles. Phys. Rev. B 2004, 69, 035417.

23. Hawa, T.; Zachariah, M. R. Coalescence Kinetics of Bare and Hydrogen-Coated Silicon Nanoparticles: A Molecular Dynamics Study. Phys. Rev. B 2005, 71, 165434.

24. Hawa, T.; Zachariah, M. R. Internal Pressure and Surface Tension of Bare and Hydrogen Coated Silicon Nanoparticles. J. Chem. Phys. 2004, 121 (18), 9043-9049.

25. Grönbeck, H.; Curioni, A.; Andreoni, W. Thiols and Disulfides on the Au(111) Surface: The Headgroup-Gold Interaction. J. Am. Chem. Soc. 2000, 122, 3839-3842.

26. Plimpton, S. J. Fast Parallel Algorithms for Short-Range Molecular Dynamics. J. Comp. Phys. 1995, 117, 1-19. 
27. Foiles, S. M.; Baskes, M. I.; Daw, M. S. Embedded-Atom-Method Functions for the FCC Metals Cu, Ag, Au, Ni, Pd, Pt, and Their Alloys. Phys. Rev. B 1986, 33 (12), 7983-7991.

28. Mei, J.; Davenport, J. W.; Fernando, G. W. Analytic Embedded-Atom Potentials for FCC Metals: Application to Liquid and Solid Copper. Phys. Rev. B 1991, 43 (6), 4653-4658.

29. Stave, M. S.; Sanders, D. E.; Raeker, T. J.; DePristo, A. E. Corrected Effective Medium Method. V. Simplifications for Molecular Dynamics and Monte Carlo Simulations. J. Chem. Phys. 1990, 93 (6), 4413-4426.

30. Raeker, T. J.; DePristo, A. E. Theory of Chemical Bonding Based on the AtomHomogeneous Electron Gas System. Int. Rev. Phys. Chem. 1991, 10 (1), 1-54.

31. Kelchner, C. L.; Halstead, D. M.; Perkins, L. S.; Wallace, N. M.; DePristo, A. E. Construction and Evaluation of Embedding Functions. Surf. Sci. 1994, 310, 425-435.

32. Lee, B.; Cho, K. Extended Embedded-Atom Method for Platinum Nanoparticles. Surf. Sci. 2006, 600, 1982-1990.

33. Haftel, M. I. Surface Reconstruction of Platinum and Gold and the Embedded-Atom Method. Phys. Rev. B 1993, 48 (4), 2611-2622.

34. Chen, S. P.; Voter, A. F.; Srolovitz, D. J. Surface Relaxation in Ordered Intermetallic Alloys. In Characterization of Defects in Materials; Siegel, R. W., Sinclair, R., Weertman, J. R., Eds.; Materials Research Society: Pittsburgh, PA, 1987; Vol. 82, p 175.

35. Jorgensen, W. L.; Madura, J. D.; Swenson, C. J. Optimized Intermolecular Potential Functions for Liquid Hydrocarbons. J. Am. Chem. Soc. 1984, 106, 6638-6646.

36. Fukunaga, H.; Takimoto, J. I.; Doi, M. A Coarse-Graining Procedure for Flexible Polymer Chains With Bonded and Nonbonded Interactions. J. Chem. Phys. 2002, $116(18), 8183-$ 8190.

37. Tries, V.; Paul, W.; Baschnagel, J.; Binder, K. Modeling Polyethylene With the Bond Fluctuation Model. J. Chem. Phys. 1997, 106 (2), 738-748.

38. Maiti, P. K.; Lansac, Y.; Glaser, M. A.; Clark, N. A. Self-Assembly in Surfactant Oligomers: A Coarse-Grained Description Through Molecular Dynamics Simulations. Langmuir 2002, 18, 1908-1918.

39 Andersen, H. C. RATTLE: A Velocity Version of the SHAKE Algorithm for Molecular Dynamics. J. Comp. Phys. 1983, 52, 24-34.

40. Rai, B.; Sathish, P.; Malhotra, C. P.; Pradip; Ayappa, K. G. Molecular Dynamic Simulations of Self-Assembled Alkylthiolate Monolayers on an Au(111) Surface. Langmuir 2004, 20, 3138-3144. 
41. Mahaffy, R.; Bhatia, R.; Garrison, B. J. Diffusion of a Butanethiolate Molecule on a Au(111) Surface. J. Phys. Chem. B 1997, 101, 771-773.

42. Beardmore, K. M.; Kress, J. D.; Gronbech-Jensen, N.; Bishop, A. R. Determination of the Headgroupgold (111) Potential Surface for Alkanethiol Self-Assembled Monolayers by Ab Initio Calculation. Chem. Phys. Lett. 1998, 286, 40-45.

43. Hautman, J.; Klein, M. L. Simulation of a Monolayer of Alkyl Thiol Chains. J. Chem. Phys. 1989, 91 (8), 4944-5001.

44. Liu, K. S. S.; Yong, C. W.; Garrison, B. J.; Vickerman, J. C. Molecular Dynamics Simulations of Particle Bombardment Induced Desorption Processes: Alkanethiolates on Au(111). J. Phys. Chem. B 1999, 103, 3195-3205.

45. Lavrich, D. J.; Wetterer, S. M.; Bernasek, S. L.; Scoles, G. Physisorption and Chemisorption of Alkanethiols and Alkyl Sulfides on Au(111). J. Phys. Chem. B 1998, 102, 3456-3465.

46. Jang, S. S.; Jang, Y. H.; Kim, Y. H.; Goddard, W. A., III; Flood, A. H.; Laursen, B. W.; Tseng, H. R.; Stoddart, J. F.; Jeppesen, J. O.; Choi, J. W.; Steuerman, D. W.; Delonno, E.; Heath, J. R. Structures and Properties of Self-Assembled Monolayers of Bistable [2]Rotaxanes on $\mathrm{Au}(111)$ Surfaces From Molecular Dynamics Simulations Validated With Experiment. J. Am. Chem. Soc. 2005, 127, 1563-1575.

47. Schmid, G. Large Clusters and Colloids. Metals in the Embryonic State. Chem. Rev. 1992, 92, 1709-1727.

48. Allen, M. P.; Tildesley, D. J. Computer Simulation of Liquids; Oxford University Press Inc.: New York, 1987.

49. Badia, A.; Cuccia, L.; Demers, L.; Morin, F.; Lennox, R. B. Structure and Dynamics in Alkanethiolate Monolayers Self-Assembled on Gold Nanoparticles: A DSC, FT-IR, and Deuterium NMR Study. J. Am. Chem. Soc. 1997, 119, 2682-2692.

50. Dalton, A. S.; Seebauer, E. G. An Improved Theory for Temperature-Dependent Arrhenius Parameters in Mesoscale Surface Diffusion. Surf. Sci. 2007, 601, 728-734.

51. Suni, I. I. The Mechanism of Surface Heterodiffusion at Elevated Temperatures. Surf. Sci. 1995, 309, L179-L183.

52. Sellers, H.; Ulman, A.; Shnidman, Y.; Eilers, J. E. Structure and Binding of Alkanethiolates on Gold and Silver Surfaces: Implications for Self-Assembled Monolayers. J. Am. Chem. Soc. 1993, 115, 530-542.

53. Thompson, S. M.; Gubbins, K. E.; Walton, J. P. R. B.; Chantry, R. A. R.; Rowlinson, J. S. A Molecular Dynamics Study of Liquid Drops. J. Chem. Phys. 1984, 81 (1), 530-542. 
54. Zachariah, M. R.; Carrier, M. J.; Blaisten-Barojas, E. Properties of Silicon Nanoparticles: A Molecular Dynamics Study. J. Phys. Chem. 1996, 100, 14856-14864.

55. Winn, J. S. Physical Chemistry; Addison Wesley: Boston, MA, 1995.

56. Lang, G. Handbook of Chemistry and Physics, 55th ed.; CRC Press: Cleveland, OH, 1974.

57. Rowlinson, J. S.; Widom, B. Molecular Theory of Capillarity; Dover Publications Inc.: Mineola, NY, 1982.

58. Dieter, G. E. Mechanical Metallurgy; McGraw-Hill Inc.: New York, 1986.

59. Yu, M.; Bovet, N.; Satterley, C. J.; Bengió, S.; Lovelock, K. R. J.; Milligan, P. K.; Jones, R. G.; Woodruff, D. P.; Dhanak, V. True Nature of an Archetypal Self-Assembly System: Mobile Au-Thiolate. Phys. Rev. Lett. 2006, 97, 166102.

60. Schreiber, F. Self-Assembled Monolayers: From 'Simple' Model Systems to Biofunctionalized Interfaces. J. Phys.: Condens. Matter 2004, 16, R881-R900.

61. Hautman, J.; Klein, M. L. Molecular Dynamics Simulation of the Effects of Temperature on a Dense Monolayer of Long-Chain Molecules. J. Chem. Phys. 1990, 93 (10), 7483-7492. 
NO. OF

COPIES ORGANIZATION

\begin{tabular}{cl}
11 & DEFENSE TECHNICAL \\
(PDF & INFORMATION CTR \\
only) & DTIC OCA \\
& 8725 JOHN J KINGMAN RD \\
& STE 0944 \\
& FORT BELVOIR VA 22060-6218 \\
1 & \\
& DIRECTOR \\
& US ARMY RESEARCH LAB \\
& IMNE ALC HRR \\
& 2800 POWDER MILL RD \\
& ADELPHI MD 20783-1197 \\
1 & \\
& DIRECTOR \\
& US ARMY RESEARCH LAB \\
& RDRL CIM L \\
& 2800 POWDER MILL RD \\
& ADELPHI MD 20783-1197 \\
1 & \\
& DIRECTOR \\
& US ARMY RESEARCH LAB \\
& RDRL CIM P \\
& 2800 POWDER MILL RD \\
& ADELPHI MD 20783-1197 \\
& \\
& ABERDEEN PROVING GROUND \\
\hline 1 & DIR USARL \\
& RDRL CIM G (BLDG 4600)
\end{tabular}


INTENTIONALLY LEFT BLANK. 